\title{
An investigation of steady flow toward a gravity well
}

\author{
BY HOWARD P. HALL ${ }^{*}$
}

Texte français, p. 36

\begin{abstract}
An investigation, by means of a series of largescale tests, of steady-state seepage in the immediate vicinity of a fully penetraiing gravity well is described, and the results presented. Particular attention is paid to the flow pattern in the region where the slope of the phreatic surface becomes so steep that Dupuit's assumptions are not valid.

The design and construction of the model are described, after which the model tests are described and the test measurements and results presented.

$A$ discussion of the relaxation method includes a description of Yang's modification of the Southwell procedure, analyses of several test cases, and a comparison of relaxation analyses with the results of model investigations of the corresponding cases. Satisfactory agreement between a relaxation analysis and the corresponding model-test results is obtained if ac-
\end{abstract}

count is taken of capillary effects. These are of particular importance near the well circumference.

A pair of empirical equations are proposed, from which to locate the phreatic line on a typical radial section through a given well.

It is concluded (1) that the relaxation method, as modified by Yang, is a valid analytical approach to problems of seepage toward a gravity well, (2) that a unique solution by this method does not follow "automatically," but requires careful checking of the solution against the boundary conditions at the phreatic surface, (3) that serious distortion of the theoretical flow pattern near the intersection of the phreatic surface and the well circumference may be produced by capillary effects, and (4) that these effects can be handled with sufficient accuracy in a relaxation analysis by introducing appropriate boundary conditions.

\section{I.-INTRODUCTION}

\section{A) Definitions}

Seepage of ground water toward a well may be idealized as a case of axially-symmetrical three-dimensional flow of a homogeneous fluid through a porous medium.

If the water-bearing stratum is not overlain by an impervious layer, the ground water stream is exposed to the atmosphere, and there exists within the ground water stream an imaginary surface at all points of which the fluid pressure is that of the atmosphere. Disregarding capillary water, this imaginary surface constitutes the upper boundary of the ground water stream, and is referred to as the phreatic surface, or the free surface. Under these circumstances, the fluid particles move under the influence of gra- vity as well as fluid head differential, and the condition which results is referred to as gravity flow. A well which drains the pervious stratum in this case is called a gravity well.

It will be observed that even after a steady state of flow toward a gravity well has been reached contact between the phreatic surface and the well occurs at an elevation above that of the water surface in the well. The circumferential surface of the well between these two elevations is referred to as the free discharge surface.

\section{B) Objectives}

Until very recently, the best available methods of analysis of steady-state seepage toward a

(*) Based on a dissertation submitted by the author in December, 1950, to the Faculty of Arts and Sciences of Harvard University.

$\left(^{*}\right)$ Assoc. Prof, of Civil Eng'g., Northwestern University, Evanston, Illinois, U.S.A. 
gravity well have been limited in their validity to regions where the slope of the phreatic surface is sufficiently small (less than about 15 per cent) to permit basing the analysis on Dupuit's fundamental assumptions [2] (*). However, with the development of Southwell's relaxation method $[4,5]$, and its adaptation, particularly in the manner devised by S. T. Yang [7], to the problem of interest here, accurate theoretical analysis of ffow conditions appears possible.

It was the purpose of the project reported in this paper to investigate steady-state scepage conditions in the vicinity of a gravity well by means of tests on a relatively large-scale model; and to establish a satisfactory correlation between the results obtained from the model tests and those derived from the theoretical analysis. Such a correlation would serve the double objective of (1) indicating that some of the more troublesome features of earlier sand model tests can be overcome, and (2) substantiating the reliability of the relaxalion procedure in a not unduly tedious and time-consuming form.

\section{II.-THE MODEL}

\section{A) Design}

1. Essential features.-The well model was constructed to represent a 15-degree sector of the region surrounding a fully penetrating gravity well. Fig. 1 is a sketch showing the essen-

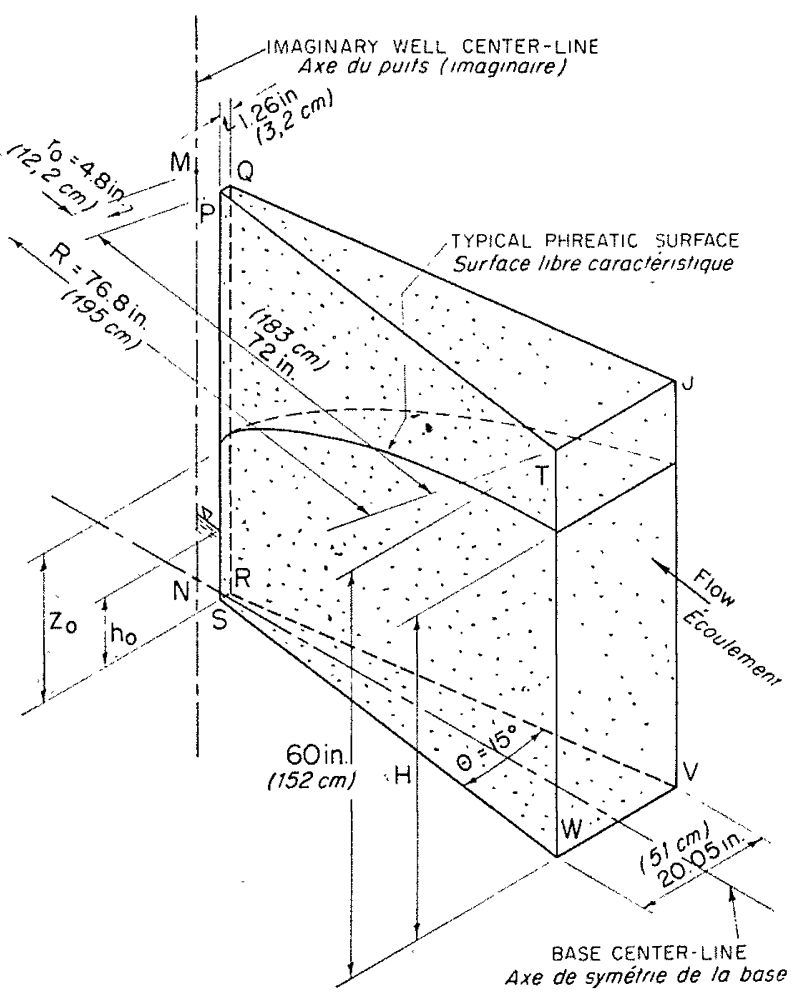

Fig. 1.--Essential dimensions of well model. Cotes principales du modèle.

tial dimensions. The side-walls, PTWS and QUVR, represent vertical radial sections through the center-line, MN, of the well; the surface

${ }^{\star}$ ) Figures in square brackets refer to the bibliography listed at the end of the paper.
PQRS, the discharge face of the model, represents a part of the circumferential surface of the well; the surface TUVW, the entrance face of the model, represents a part of a vertical cylindrical surface surrounding the well at some distance from it; and the surface SRVW represents an underlying horizontal impervious base. Thus the distance $\mathrm{MT}(=\mathrm{MU}=\mathrm{NW}=\mathrm{NV})$ corresponds to the radial distance, $R$, from the well center-line to a point of zero or negligible drawdown; and the distance $\mathrm{MP}(=\mathrm{MQ}=\mathrm{NS}=\mathrm{NR})$ corresponds to the well radius, $r_{0}$.

2. Proportioning.--Large size was desirable to facilitate observations within a distance of a few radii of the well circumference, and to minimize the importance of capillary action. A low value of 0 (fig. 1) was desirable in order to justify the use of plane entrance and discharge faces as simpler substitutes for the cylindrical surfaces to be simulated. On the other hand, the discharge face width (PQ or SR in Fig. 1) had to be large enough so that ordinary tolerances in machining would not introduce appreciable irregularities; and the value of the ratio $\mathrm{R} / r_{0}$ had to be large enough so that the vertical entrance face would be a reasonable approximation of the equipotential surface at a corresponding distance from a typical well.

A value of $15^{\circ}$ was chosen for $\theta$, and a ratio of 16 to 1 for $\mathrm{R} / r_{0}{\left({ }^{\star *}\right)}^{*}$. The radius of the well thus became $r_{0}=4.80$ inches, and the distance from well center-line to entrance face became $\mathrm{R}=76.80$ inches. The clear distance between side-walls under these conditions was 1.26 inches at the discharge face and 20.05 inches at entrance.

(**) According to the results of BABBrTr and CALDWELL [1], equipotential surfaces are nearly vertical cylinders at a distance of between fifteen and twenty times the well radius. 


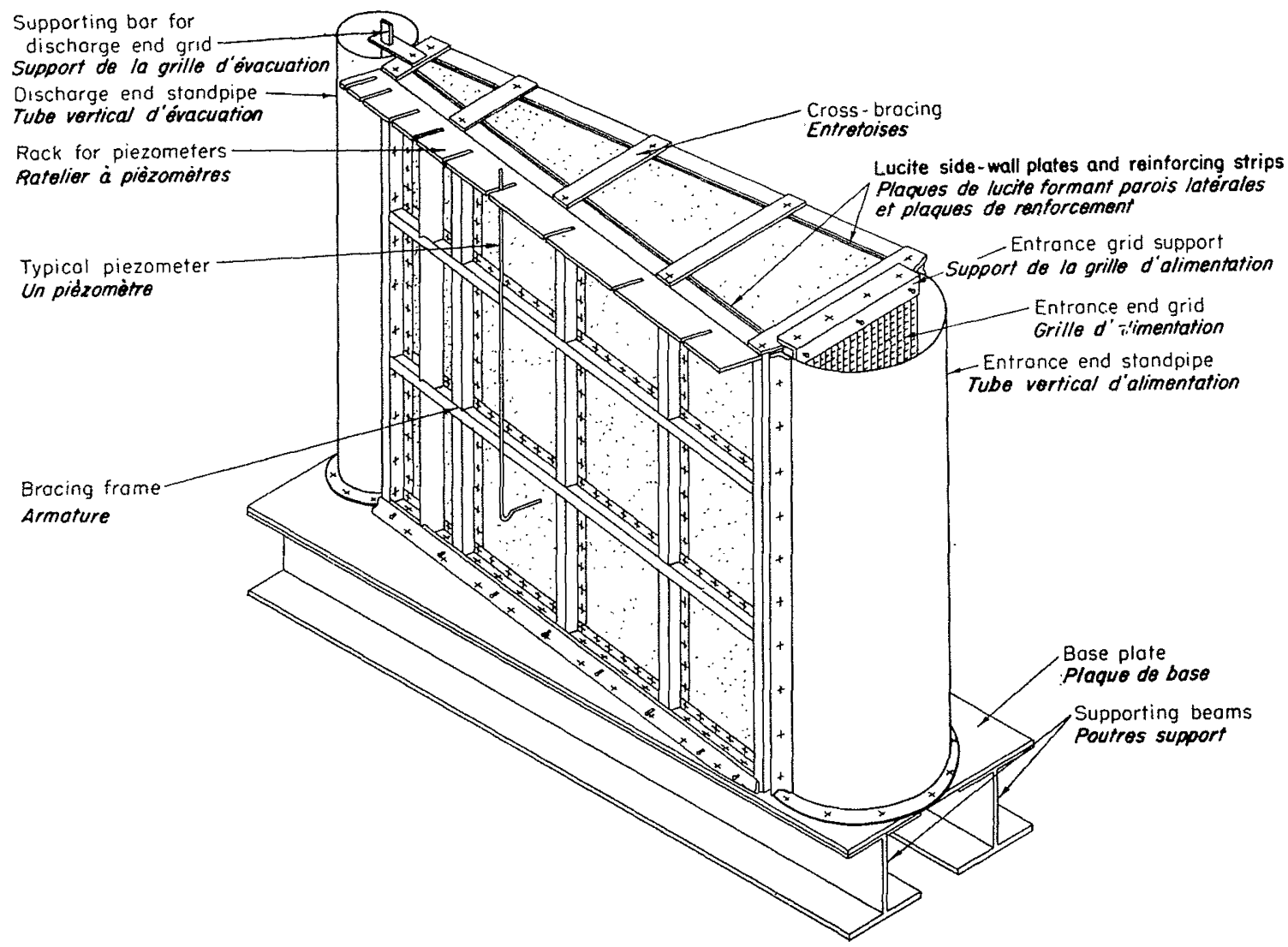

FIG. 2.-Isometric drawing of well model.

Vue perspective du modèle.

3. Porous medium.--Standard Ottawa $\left(^{*}\right)$ sand was selected as the porous medium which offered the best combination of economy and desirable physical properties. The high degree of uniformity of grainsize and the nearly spherical grain-shape reduced the importance of errors resulting from non-homogeneity and stratification.

4. Gradient and rate of discharge.-An estimate of average gradient at the discharge face was obtained from the Dupuit-Thiem equation. Assuming for this purpose that $h_{0}=0$ and $Z_{0}=(0.5) \times(H)=2$ feet, the average gradient at the discharge face was computed to be about 3.5. Tests performed on Ottawa sand to determine the range of validity of Darcy's Law indicated a slight deviation from direct proportionality between discharge velocity and gradient at a value of gradient of approximately 3.0. The sand was tested at about the same void ratio as that at which it was to be placed in the model. Using the Dupuit-Thiem equation with $k=0.4 \mathrm{~cm} / \mathrm{s}$, the anticipated maximum rate of discharge was computed to be about $250 \mathrm{~cm}^{3 /} / \mathrm{s}$.

5. Detarls.-Fig. 2 is an isometric drawing

$\left(^{\star}\right)$ Ottawa is a small lown in the state of minois (U.S.A.). of the model. For the sake of clearness, only one piezometer is shown, and no details of the waler circulation system are included.

Transparent side-walls were desirable to permit observation of conditions at the free surface; and lucite was preferred to glass because it was easier to handle and to machine.

To prevent excessive deflection of the lucite side-walls, steel bracing frames were designed to bear against them. The bracing frames were fastened to the base plate at the bottom and to each other across the top of the model.

A steel base plate 1/4-inch in thickness, supported by two heavy wide-flange beams was considered adequate to prevent excessive deflection.

At the entrance and discharge faces plastic screens were preferred to metal to reduce danger of electrolytic action or rusting.

A heavy standpipe at the discharge end was desirable to increase the rigidity of the structure at a point where deflections would be most troublesome. At the entrance end an approximately semi-cylindrical thin sheet metal standpipe was satisfactory. A larger cross-section was desirable in this case to permit inflow at the required maximum rate without the possibility of turbulence at the entrance surface. 


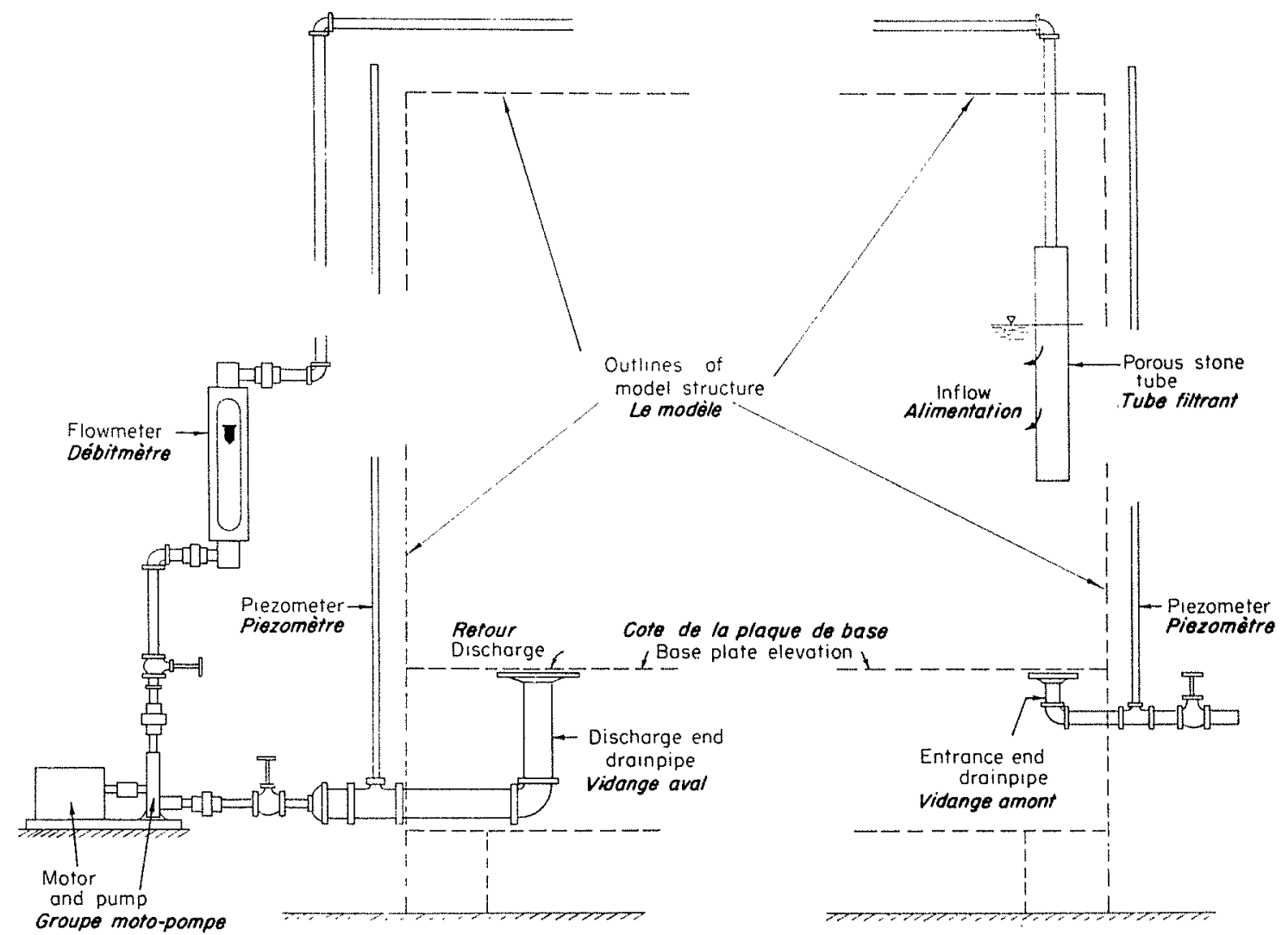

FIG. 3.-Diagram of system for circulation of water.

Schéma du circuit d'eau.

A closed system of water circulation was desirable in order to hold down the quantity of air which would be introduced during test runs. The circulation system. (Fig. 3) included a pipe leading from the discharge standpipe to a centrifugal pump, thence through a flow-measuring device and back into the entrance reservoir through a porous stone tube intended to reducc turbulent effects.

Connections and seals were potentially the most troublesome detail. Rubber gaskets were chosen as the most satisfactory type of seal.

\section{B. Construction and Preparation for Tests}

The details of the construction are shown in Figs. 4-9.

The model was first filled with warm water. The object was to keep the water in the model warm until shortly before beginning the tests, so that when it was finally allowed to cool, it would be deficient in dissolved air when it reached room temperature. Thus, as tests proceeded, the water would tend to pick up air, rather than release it to form air voids.

After the model had been filled with water, the sand was saturated in small quantities and placed under water.

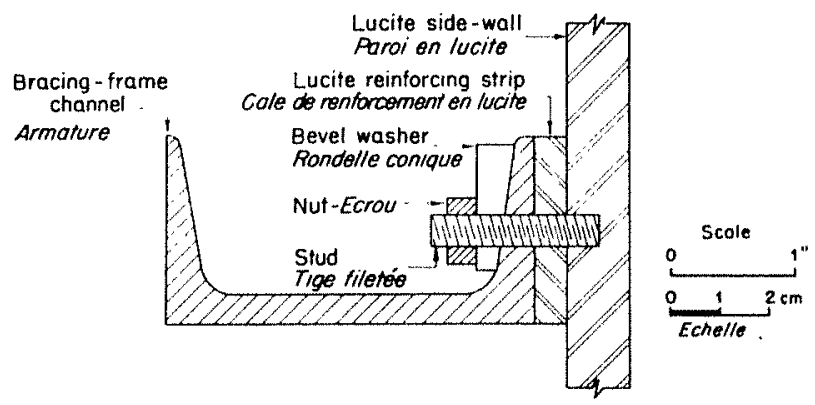

a) Typical connection between side-wall and bracing frame.

a) Liaison entre les parois et une armalure.

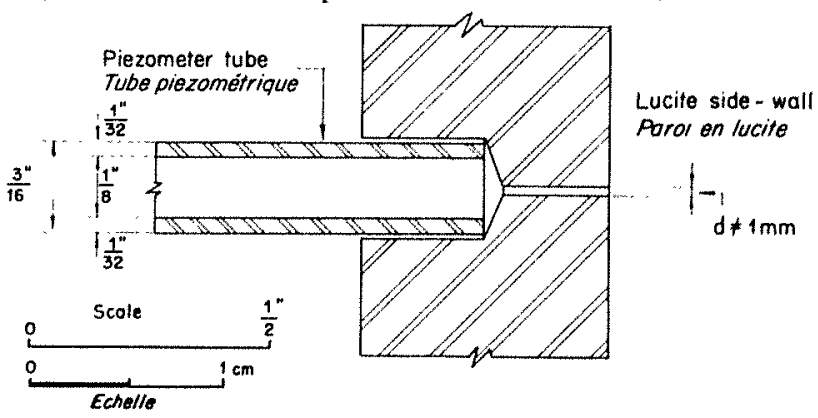

b) Typical piezometer connection.

b) Une prise de pression.

Fri. 4.-Well model details. Détails da modète. 


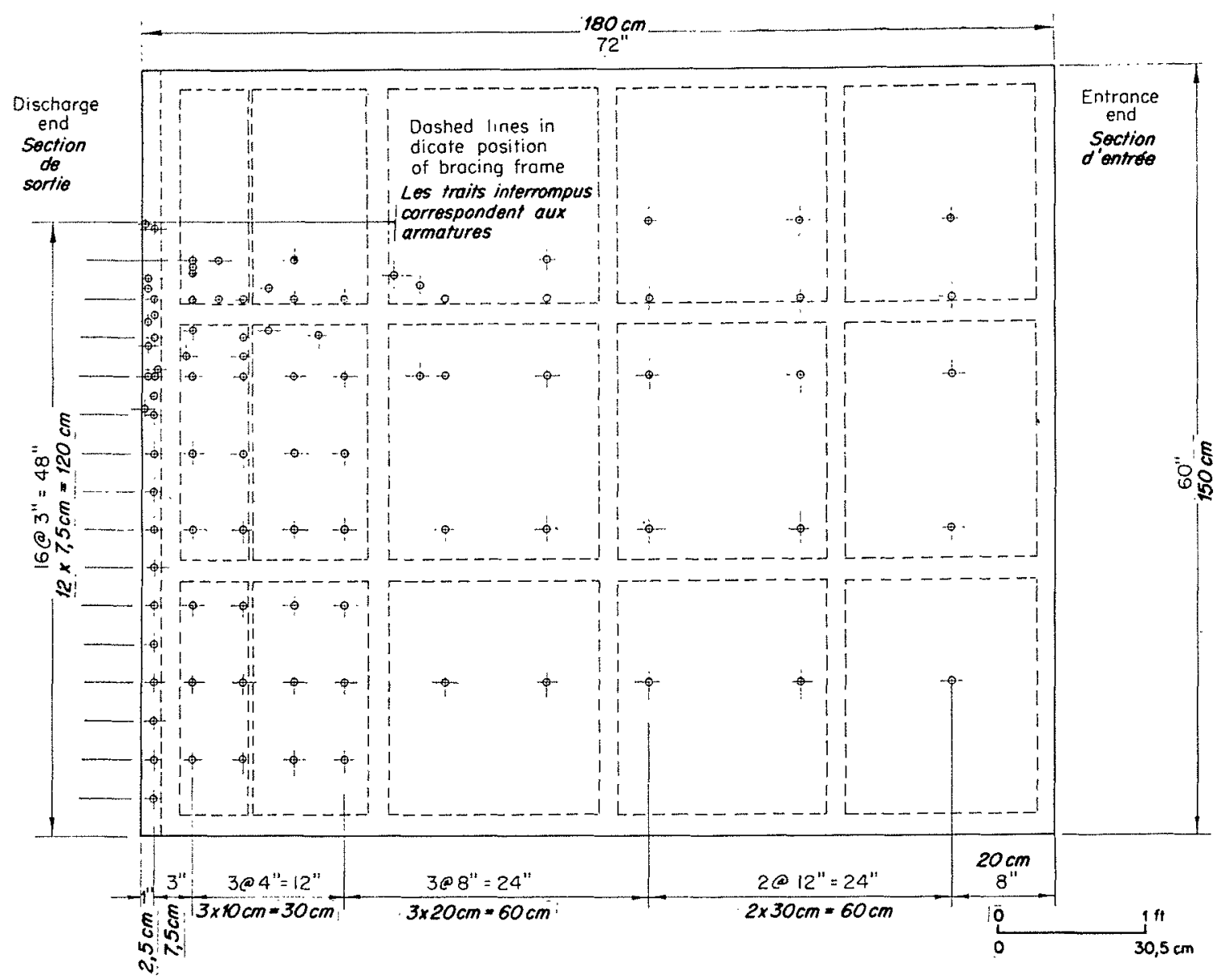

Fig. 5.-Elevation of lucite side-wall.

Vue de face des panneaux en lucite.

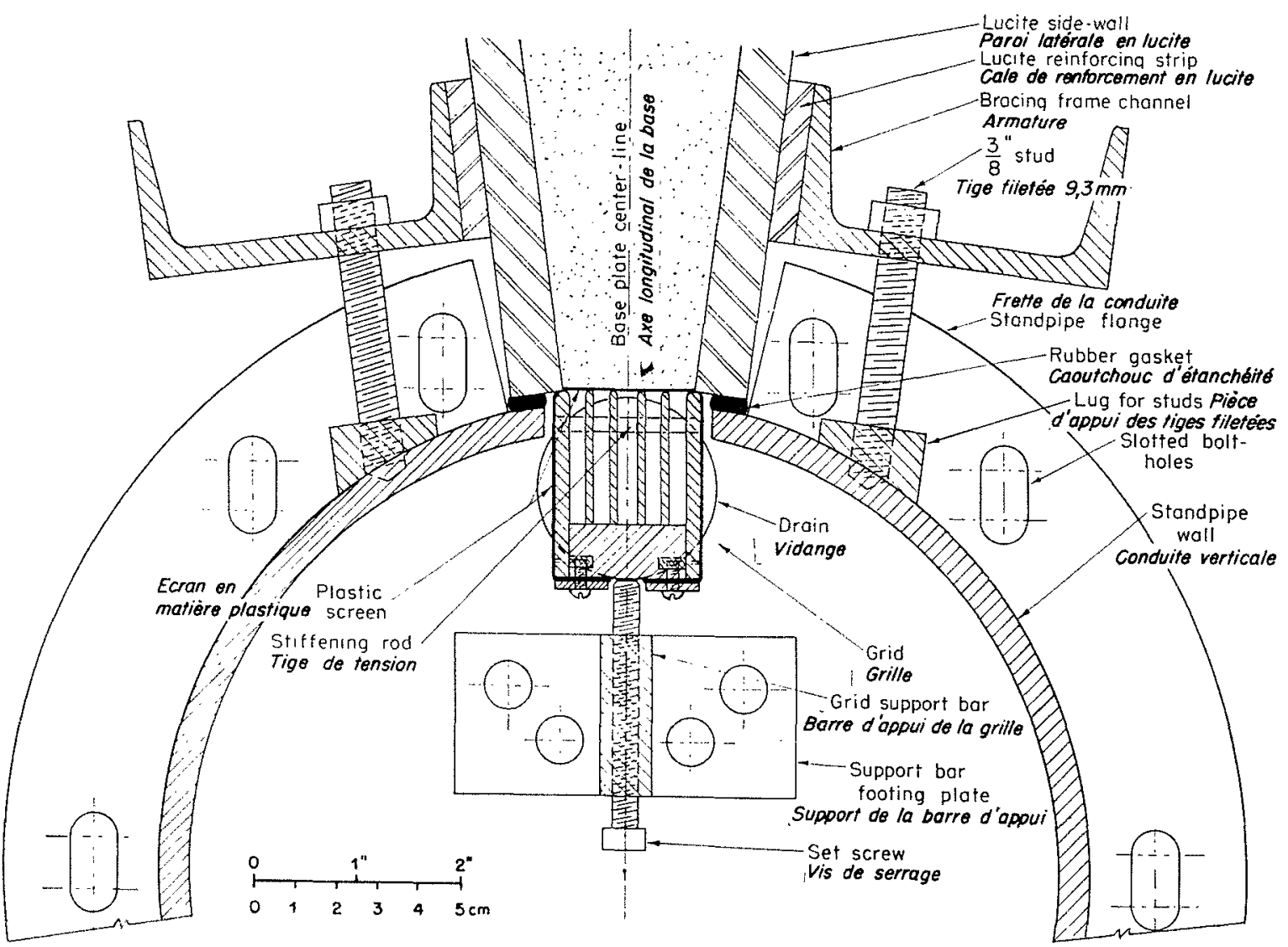

Fig. 6.-Horizontal section through discharge surface.

Coupe horizonfale à travers la section de sortie. 


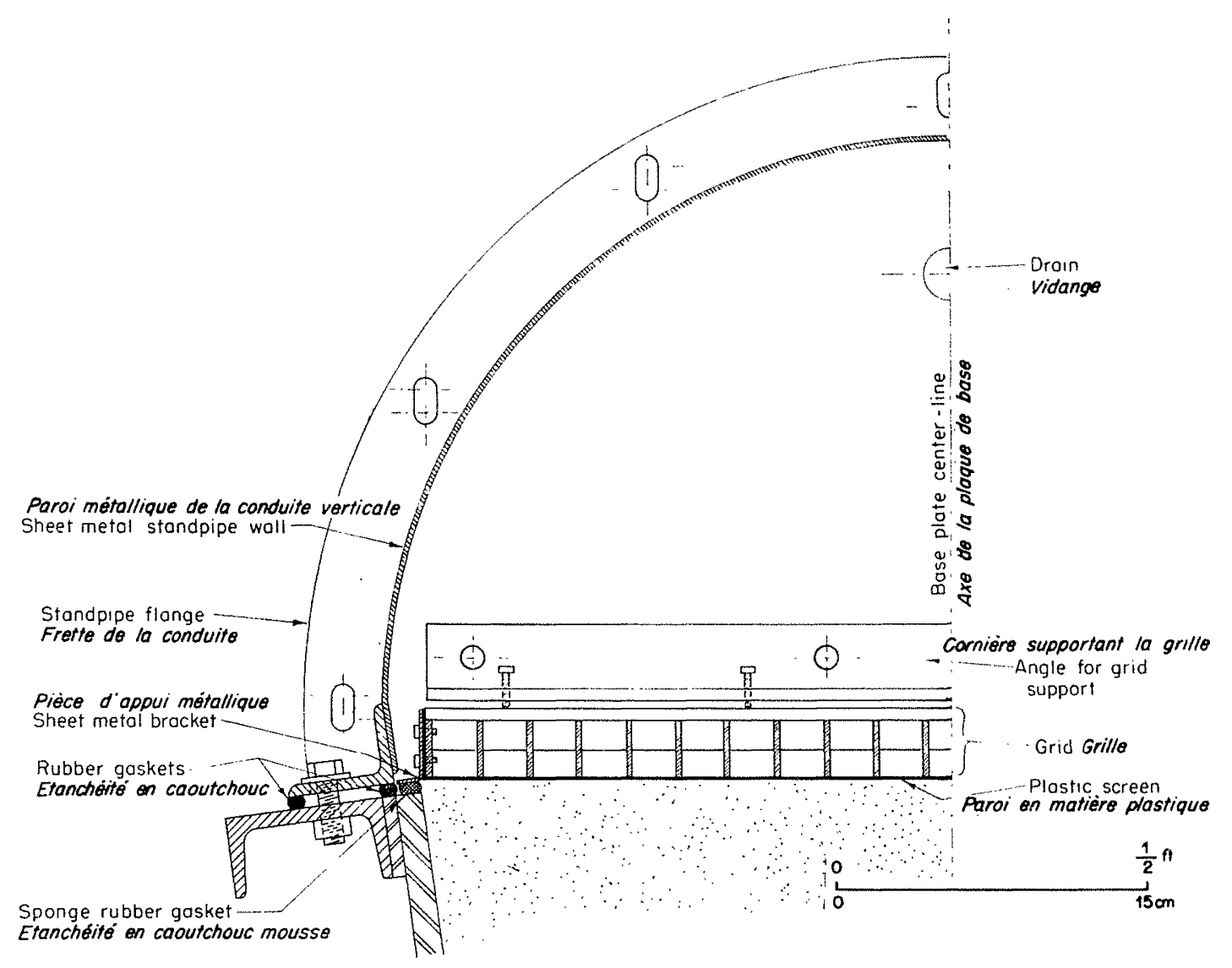

FIa. 7. - Horizontal section througl entrance face.

Coupe horizontale à travers la section d'entrée.

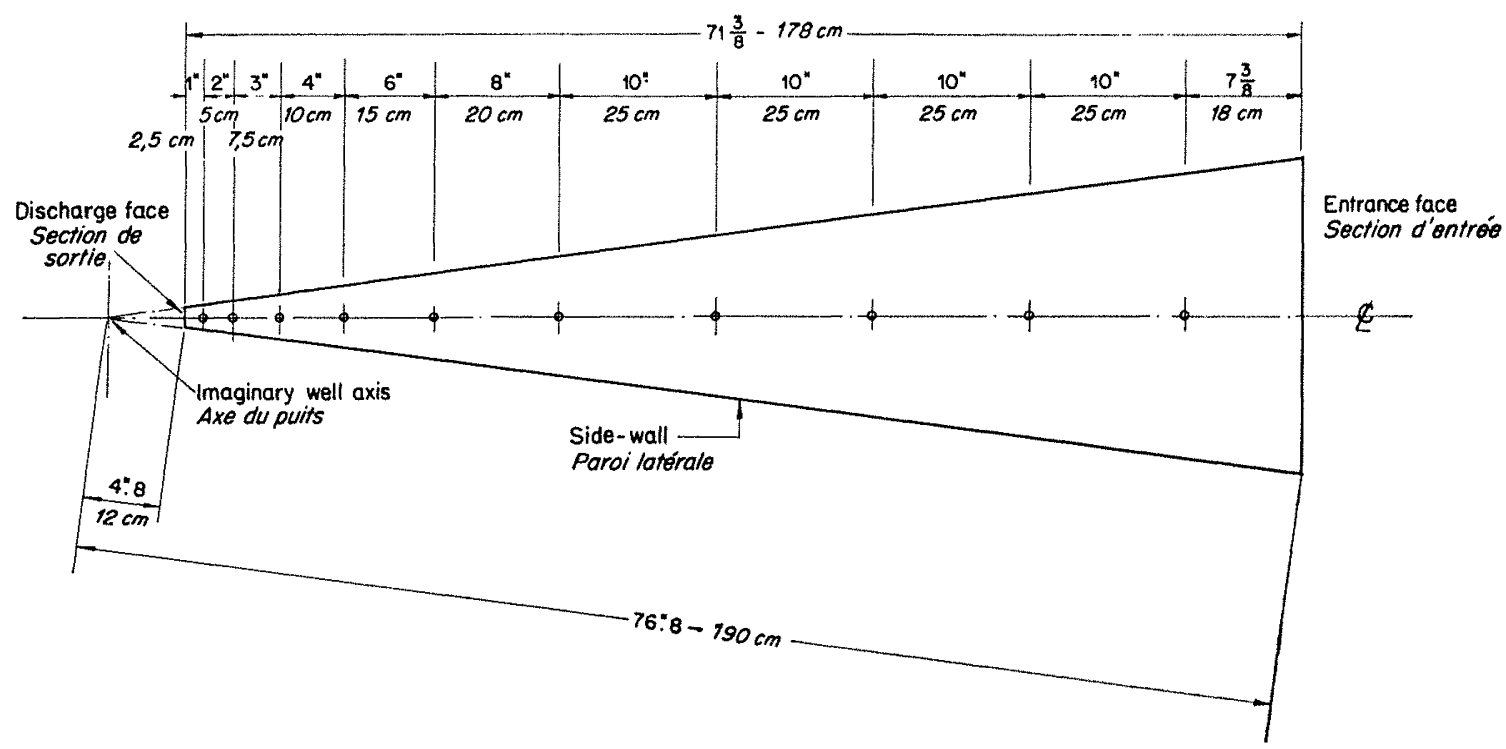

Fici. 8,-focation of base-plate piezometer openings.

Disposition des orifices piézométriques sur le fond. 


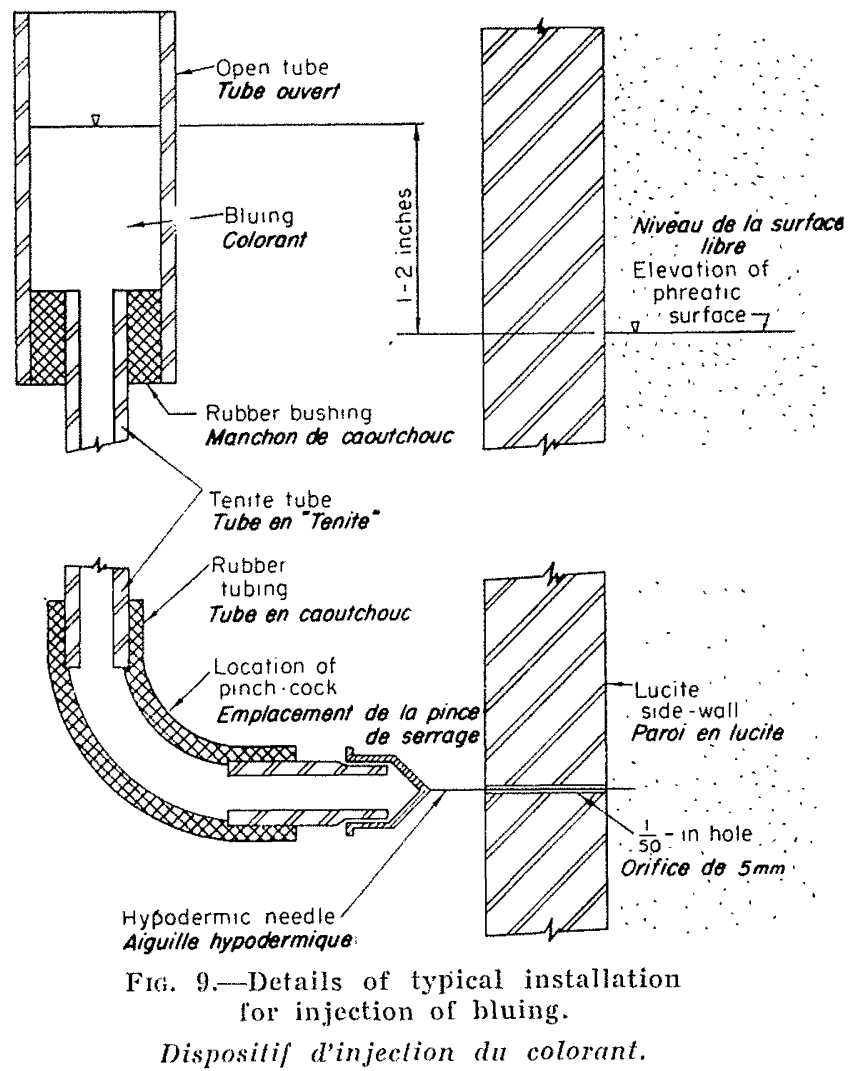

III.-MODEL TESTS

\section{A) Performance}

1. Obuectives.-The chief objective of the model tests was the determination of the steadystate pattern of flow toward a gravity well within the region represented by the model. Other objectives included an investigation of the validity of Darcy's Law for computing the rate of discharge, the determination of the effect of a capillary layer on the rate of discharge and the flow pattern, and an investigation of the effects of degree and uniformity of saturation of the sand.

2. Characteristic ratios.- The flow pattern is a function of the four independent variables, $\mathrm{R}, r_{0}, \mathrm{H}$, and $h_{0}$ (see Fig. 1). Thus, a particular problem may be defined by the values of any three independent ratios, called the characteristic ratios, involving these variables.

In the present case, the variables were grouped into the ratios $\mathrm{R} / r_{0}, \mathrm{H} / r_{0}$, and $h_{0} / \mathrm{H}$. Of these three, the value of the first was $\mathrm{R} / r_{0}=16$ for all tests. The second ratio, $\mathrm{H} / \mathrm{r}_{0}$, was held constant at a value of about 10 by maintaining a constant water level in the entrance standpipe. The third ratio, $h_{0} / \mathrm{H}$, was set at a different value for each test within a series by adjustment of the water level in the discharge standpipe. The entire possible range, from 0 to 1 , was covered in the tests.

3. Test program.-Four series of tests werc performed. Series A consisted of eight tests following placement of the sand as noted in paragraph II.B.2. The value of $H$ was held at 48.0 inches, and $h_{0}$ was reduced by successive 6 -inch decrements from a value of 42.0 inches in the first test to a value of zero in the last.

Following Series A, the model was drained and refilled with water without removing the sand. Series B consisted of three tests run during refilling. The value of $H$ was again held at 48.0 inches, and $h_{0}$ was set successively at zero, 12.0, and 24.0 inches.

Series G repeated the tests of Series B in reverse order following complete filling of the model.

Series D was a repetition of Series $C$ with the original water replaced by water whose surface tension had been reduced by chemical treatment.

\section{B) Test Measurements}

1. Tabulation.-Table I contains a summary of the important measurements made during the tests, except for the piezometer readings. The column of values of $z_{0}$, the elevation of the point of intersection of phreatic line and well circumference, is included for completeness, although the values were determined from plots of piezometer readings rather than by direct observation.

2. Streamlines.-Figs. 10 and 11 are photographs of dye-stream lines taken during Test 8 of Series A. Plots of some of these lines also appear in Fig. $15\left(^{*}\right)$. Observed dye-stream lines for test A-6 are plotted in Fig. 14. Figs: 10, 11, 14 , and 15 show a distinct reverse curvature of the flow lines. All are slightly concave upward near the discharge face. It will be noted that the uppermost flow line of Test A-8 (Fig. 15), whose course is well within the capillary layer, breaks sharply near the discharge face and passes below the phreatic line.

Table II contains measurements made for determining the relative relocities of streamlines. For the most part the time readings are accurate to within about a tenth of a minute. However, the degree of accuracy decreases toward the dis-

(*) The grid upon which ligss, 12-16, 22-25 and 27 are plotted represents a 2 -inch square grid drawn on the side-wall plates of the model. 
TABLE I.-TEST PROGRAM ANI) SUMMARI\%ED DATA

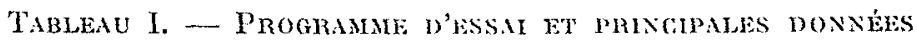

\begin{tabular}{|c|c|c|c|c|c|c|c|c|c|c|c|c|}
\hline \multirow{3}{*}{ 兽芒 } & \multirow{3}{*}{ 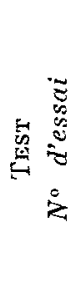 } & \multicolumn{4}{|c|}{$\begin{array}{l}\text { CONTROLLED DATA } \\
\text { Grandeurs réglées }\end{array}$} & \multicolumn{6}{|c|}{$\begin{array}{l}\text { Mrasured Data } \\
\text { Grandents mesurées }\end{array}$} & \multirow{3}{*}{$\begin{array}{l}\text { Remanks } \\
\text { Observations }\end{array}$} \\
\hline & & \multicolumn{2}{|c|}{$\mathrm{H}$} & \multicolumn{2}{|c|}{$h_{0}$} & \multicolumn{2}{|c|}{$\mathrm{Z}_{0}$} & \multicolumn{2}{|c|}{$\begin{array}{l}\text { Ave. } h_{c} \\
\text { (moy.) }\end{array}$} & \multirow{2}{*}{$\begin{array}{c}\mathrm{T} \\
(" \mathrm{C})\end{array}$} & \multirow{2}{*}{$\begin{array}{c}0 \\
\mathrm{ec} / \mathrm{s} \\
\mathrm{cm} 3 / \mathrm{s}\end{array}$} & \\
\hline & & (in.) & $(\mathrm{cm})$ & (in.) & $(\mathrm{cm})$ & (in.) & $(\mathrm{cm})$ & (in.) & $(\mathrm{cm})$ & & & \\
\hline \multirow{8}{*}{ A } & 1 & 48 & 122 & 42 & $\mid 106.4$ & 43.6 & $110.7 \mid$ & 3.5 & 8.9 & 26.8 & 83 & \multirow{8}{*}{ 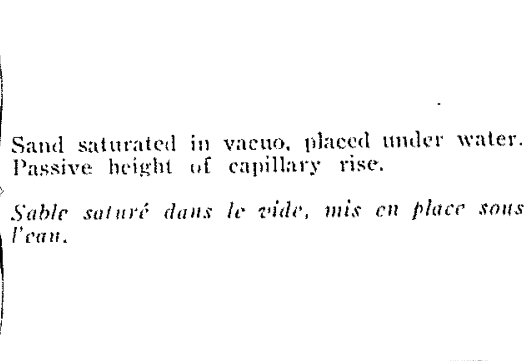 } \\
\hline & 2 & - & - & 36 & 91.2 & 41.1 & 104.3 & - & -1 & 28.5 & 161 & \\
\hline & 3 & - & 1 & 30 & 76 & 37.2 & 94.5 & - & -1 & 29.0 & 227 & \\
\hline & 4 & $\cdots$ & $\cdots$ & 24 & 60.8 & 35.5 & 90.2 & $\cdots$ & $\cdots$ & 29.1 & 277 & \\
\hline & 5 & - & $1 \cdots$ & 18 & 45.6 & 34.0 & 86.4 & - & $\cdots$ & 29.5 & 319 & \\
\hline & 6 & - & $1-$ & 12 & 30.4 & 33.4 & 84.8 & - & $\ldots-$ & 29.2 & 345 & \\
\hline & 7 & - & - & 6 & 15.2 & 32.8 & 83.3 & $\cdots$ & $\cdots$ & 29.5 & 366 & \\
\hline & 8 & $\cdots$ & $\cdots-$ & () & 0 & 32.8 & 83.3 & $\cdots$ & $\cdots \cdots$ & 29.6 & $\cdots$ & \\
\hline \multirow{3}{*}{ B } & 1 & 47.5 & 120,6 & 0 & 0 & 33.9 & 86.1 & 2.75 & 7 & 31.4 & $\cdots$ & \multirow{3}{*}{ 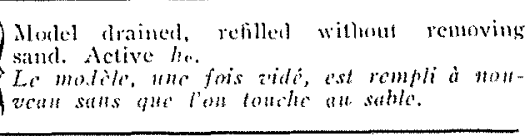 } \\
\hline & 2 & - & - & 12 & 30.4 & 35.4 & 89.9 & - & - & 31.6 & $207-265$ & \\
\hline & 3 & - & - & 24 & 60.8 & 35.8 & 90.9 & 3.0 & 7.6 & 31.8 & $311-248$ & \\
\hline \multirow{3}{*}{ C } & 1 & - & - & 24 & 60.8 & 34.1 & 86.6 & 4.0 & 10.2 & 26.2 & 265 & \multirow{3}{*}{$\begin{array}{l}\text { Reversal of tests of Series B. Passive he. } \\
\text { Reprise des essais de la serie B, an seHs } \\
\text { inversc. }\end{array}$} \\
\hline & 2 & - & - & 12 & 30.4 & 32.4 & 82.3 & - & - & 26.2 & 320 & \\
\hline & 3 & - & - & 0 & 0 & 32.0 & 81.31 & 4.5 & 11.4 & 25.7 & 341 & \\
\hline \multirow{3}{*}{ D } & 1 & - & - & 24 & 60.81 & 33.7 & 85.6 & 1.5 & 3.8 & 25.0 & 242 & \multirow{3}{*}{ 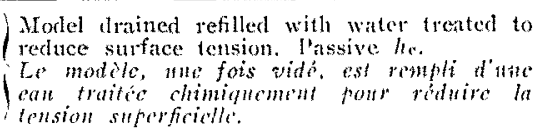 } \\
\hline & 2 & - & - & 12 & 30.4 & 31.9 & 81 & - & $-\ldots$ & 25.0 & 292 & \\
\hline & 3 & - & $1 \ldots$ & 0 & 0 & 31.4 & 79.8 & - & $\cdots$ & 25.0 & $\cdots$ & \\
\hline
\end{tabular}

DATA COMMON TO ALI, TESTS

Données communes à tous les essuts

$$
\mathrm{R}=76.8 \mathrm{in} .(197,6 \mathrm{~cm})
$$

$r_{0}=4.8 \mathrm{in} .(12.2 \mathrm{~cm})$

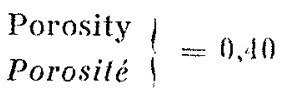

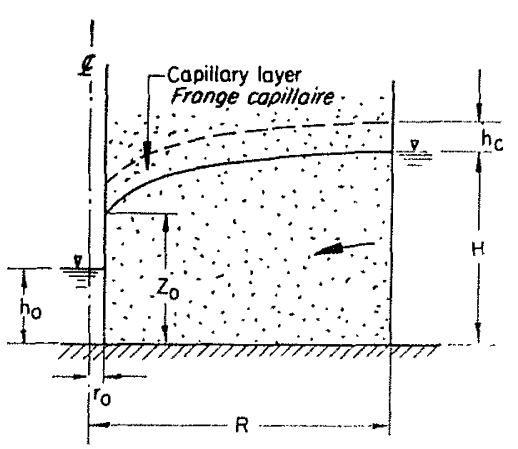

Key : radial section

Section radiale : schéma des notalions charge end, since the dyc-streams became less dense and therefore more difficult to detect as their velocity increased.

3. Piezometer Readings.-The piezomeler readings formed the largest part of the test measurements. In Figs. 12-15 each piezometer reading is recorded at its location on the lucite side-wall. The phrealic lines were plotled from the piezometer readings, and the upper boundary of the capillary layer was located from direct observations.

\section{C) Test Results}

1. General.-The degree of saluration was greatest in Series $A$. This was the only case in which the water was introduced into the model before the sand and the sand placed under water after having been salurated in small loads under: a vacuum. The amount and distribution of air voids did not become an important sourec of error in Series A until the final test.

In Series B, and to a progressively lesser extent in Series $G$ and $D$, the most serious disturb- 


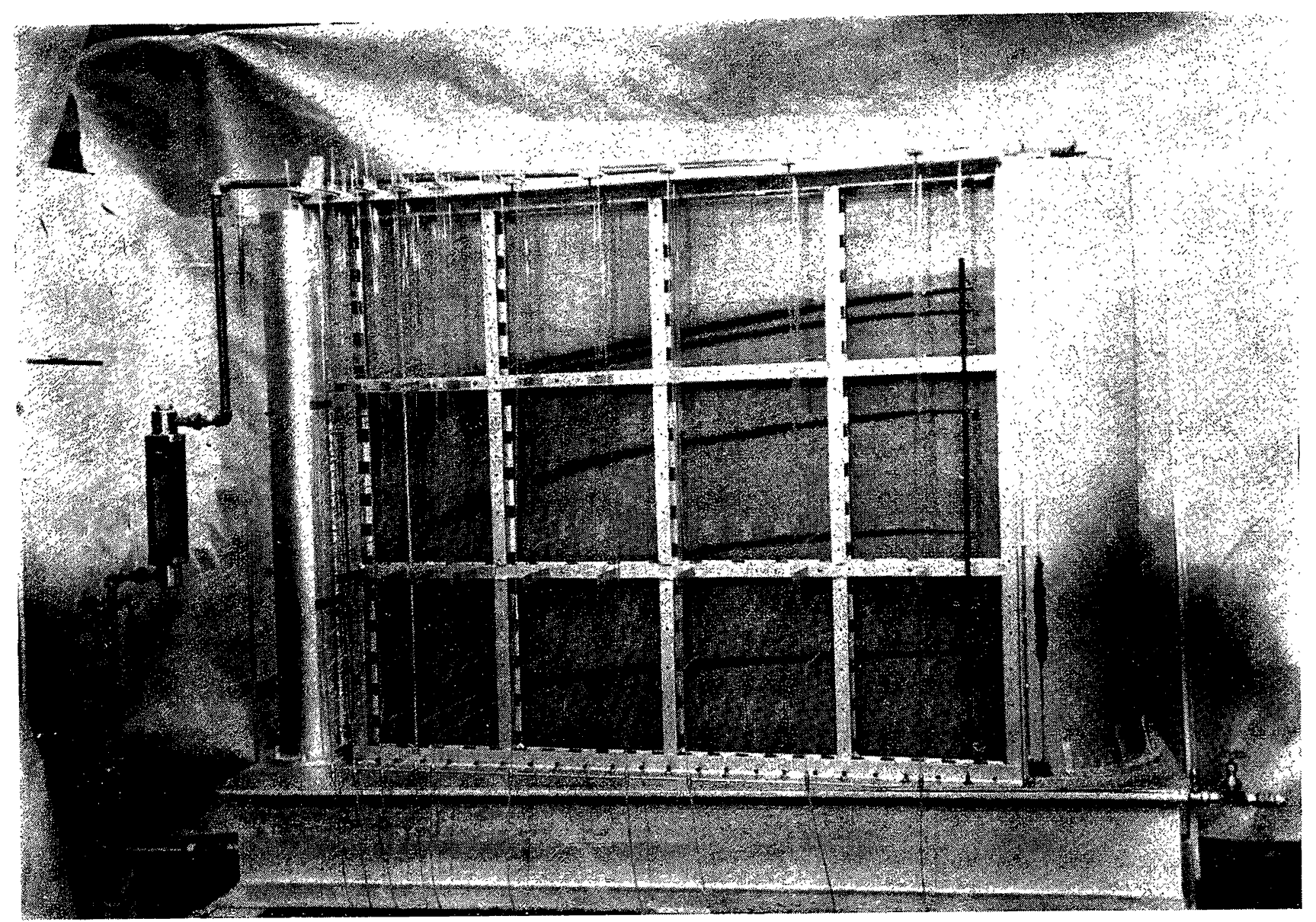

F16. 10.-Series A, test 8: Dye-stream lines : first day.

Serie A, essai 8 : Les lignes de conrant colorees (10r jour).

TABLE II.-SERIES A, TEST 8; STREAMLINE VELOCITY DATA

Tableau II. - Série A, essai 8 ; Mesures de vitesse des ligives de courant

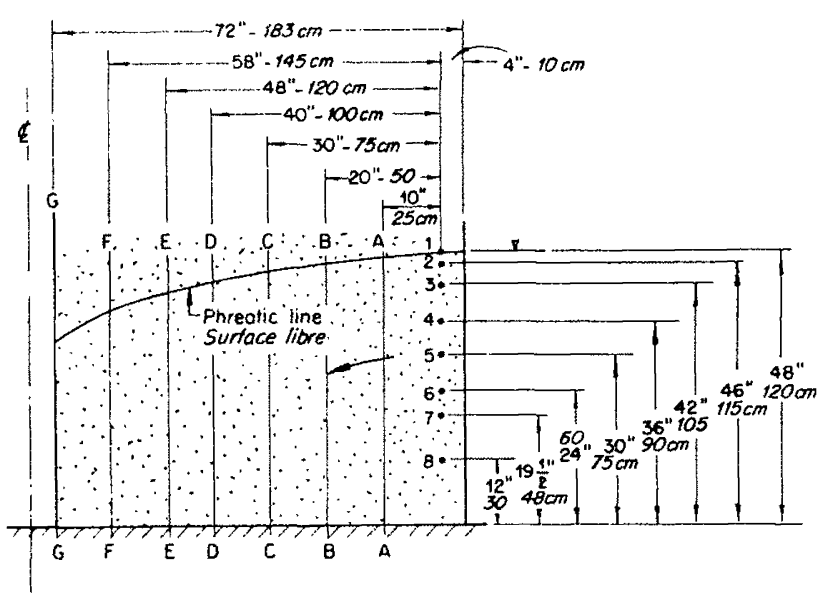

\begin{tabular}{|c|c|c|c|c|c|c|c|c|}
\hline \multirow{2}{*}{$\begin{array}{c}\text { PoiNT } \\
\text { OF } \\
\text { INJECTION } \\
\text { Point } \\
\text { d'injection }\end{array}$} & \multicolumn{7}{|c|}{$\begin{array}{l}\text { THME, IN MINUTES, REQUIRED TO REACH SECTION } \\
\text { T'emps, en minutes, nécessaire pour atteindre } \\
\text { la section }\end{array}$} & \multirow{2}{*}{ 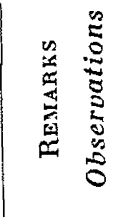 } \\
\hline & $A-A$ & $\mathrm{~B}-\mathrm{B}$ & $\mathrm{C}-\mathrm{C}$ & $\mathrm{D}-\mathrm{D}$ & E-E & $\mathrm{F}-\mathrm{F}$ & $\mathrm{G}-\mathrm{G}$ & \\
\hline 1 & 2.8 & 6.0 & 9.0 & 11.3 & 13.5 & 15.0 & $\overline{16.0}$ & \multirow{8}{*}{$\begin{array}{l}\text { Capillary } \\
\text { layer. } \\
\text { Frangc } \\
\text { capillaire. }\end{array}$} \\
\hline 2 & 2.2 & 4.5 & 6.7 & 8.5 & 10.1 & 11.2 & 12.0 & \\
\hline 3 & 2.0 & 4.2 & 6.2 & 8.0 & 9.3 & 10.3 & 11.0 & \\
\hline 4 & 2.0 & 4.0 & 6.2 & 7.8 & 9.0 & 10.0 & 10.5 & \\
\hline 5 & 2.0 & 3.9 & 5.8 & 7.3 & 8.4 & 9.3 & $\overline{9.8}$ & \\
\hline 6 & 1.8 & 3.8 & 5.5 & 7.0 & 8.0 & 8.8 & 9.2 & \\
\hline 7 & 1.8 & 3.6 & 5.2 & 6.8 & 7.7 & 8.4 & 9.0 & \\
\hline 8 & 1.8 & 3.5 & 5.0 & 6.6 & 7.6 & 8.0 & 8.5 & \\
\hline
\end{tabular}

ing factor was non-uniform distribution of air voids. This condition was largely eliminated in Series $C$ and $D$; but data obtained in Series B indicale that the results are of little value except
Lo show that even in the case of a large model and a porous medium as coarse as Oltawa standard sand, a condition of approximately uniform effective permeability cannot be obtained by the 


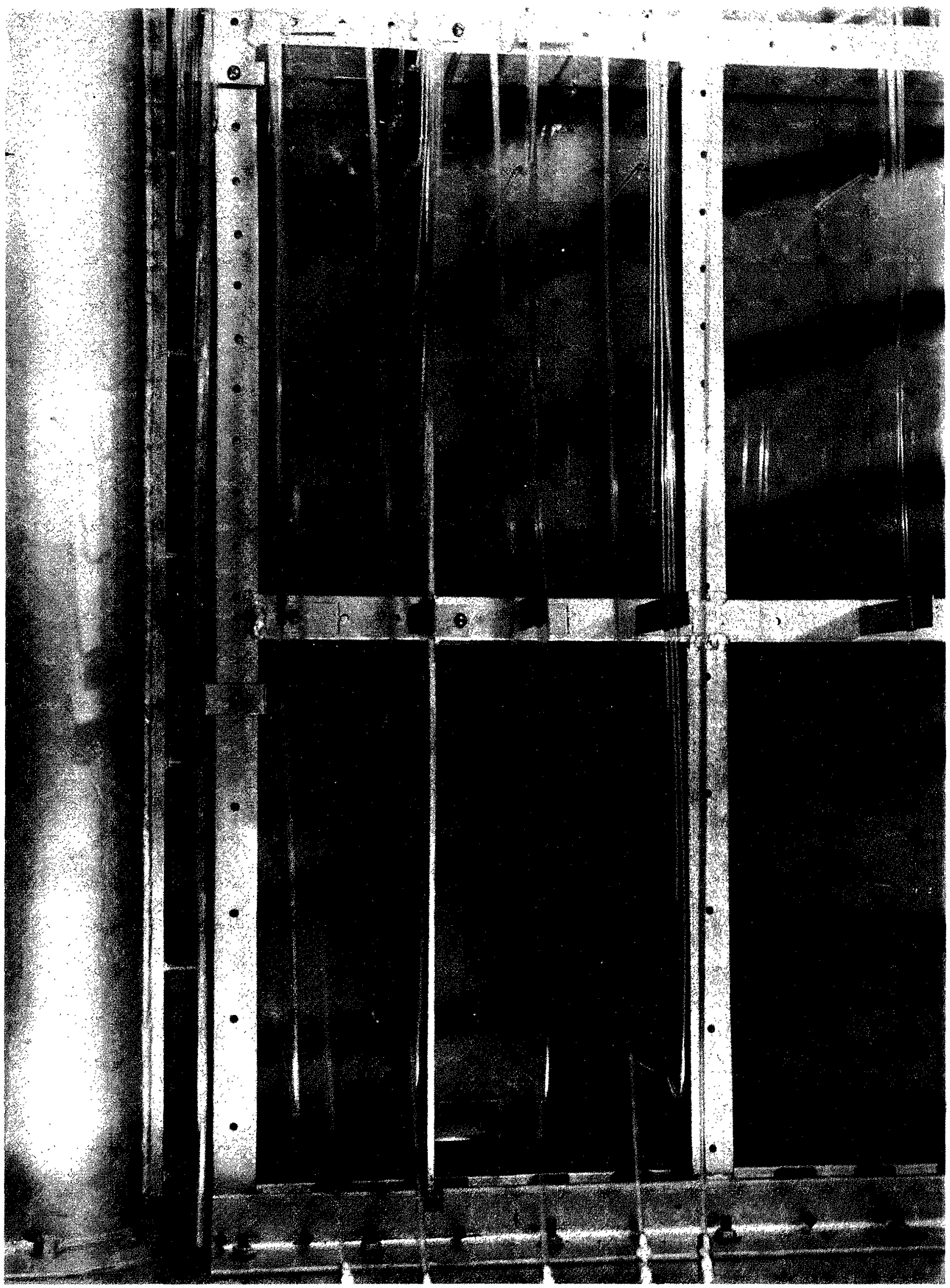

Fici, 11.--Series $d$, test 8 : Dye-stream lines: third day : detail.

Série A, essai 8: Les lignes de courant rolories (3 jour, détails). 
direct introduction of water into a mass of sand, without additional precautionary measures.

Conditions during Series A were most nearly ideal. Consequently, the results of Tests 2, 4, and 6 of Series A were selected as the basis of comparison with theoretical analyses; and the results of Series $A$ in general are used as the basis for all discussions to follow. Comparisons of the results of Series B, C, and D with those of Series A serve to illustrate the relative importance of such factors as degree of saturation, capillary action, and effective uniformity of the sand.

2. Flow Patrerns,--Figs. 12-14 show the flow patterns for Tests 2, 4, and 6, respectively, of Series A, as determined from test observations. In each case, a set of equipotential lines dividing the permeated region into ten equal potential intervals is located by interpolation from the piezometer readings. Thus, the potential intervals are respectively, 1.2, 2.4, and 3.6 inches of water in the three tests. The phreatic line in each case was determined by locating the point of intersection of each equipotential line and the corresponding level of elevation, and drawing a curve through the resulting series of points. The flow lines of Fig. 14 are plots of the paths followed by streams of bluing injected at the indicated points.

Fig. 15 provides the same information for Test 8 of Series A as Figs. 12-14 for Tests 2, 4, and 6 . No changes in piezometer readings were observed in progressing from Test 7 to. Test 8 until more than a day had elapsed. After two days a second set of piezometer readings for Test 8 showed a general increase. The largest increases in individual piezometer readings were noted in the lower corner at the discharge face. This increase was apparently attributable to the formation of air voids in that region. The non-uniform character of this condition was indicated by the fact that a corresponding increase was not observed elsewhere. No measurable change in the

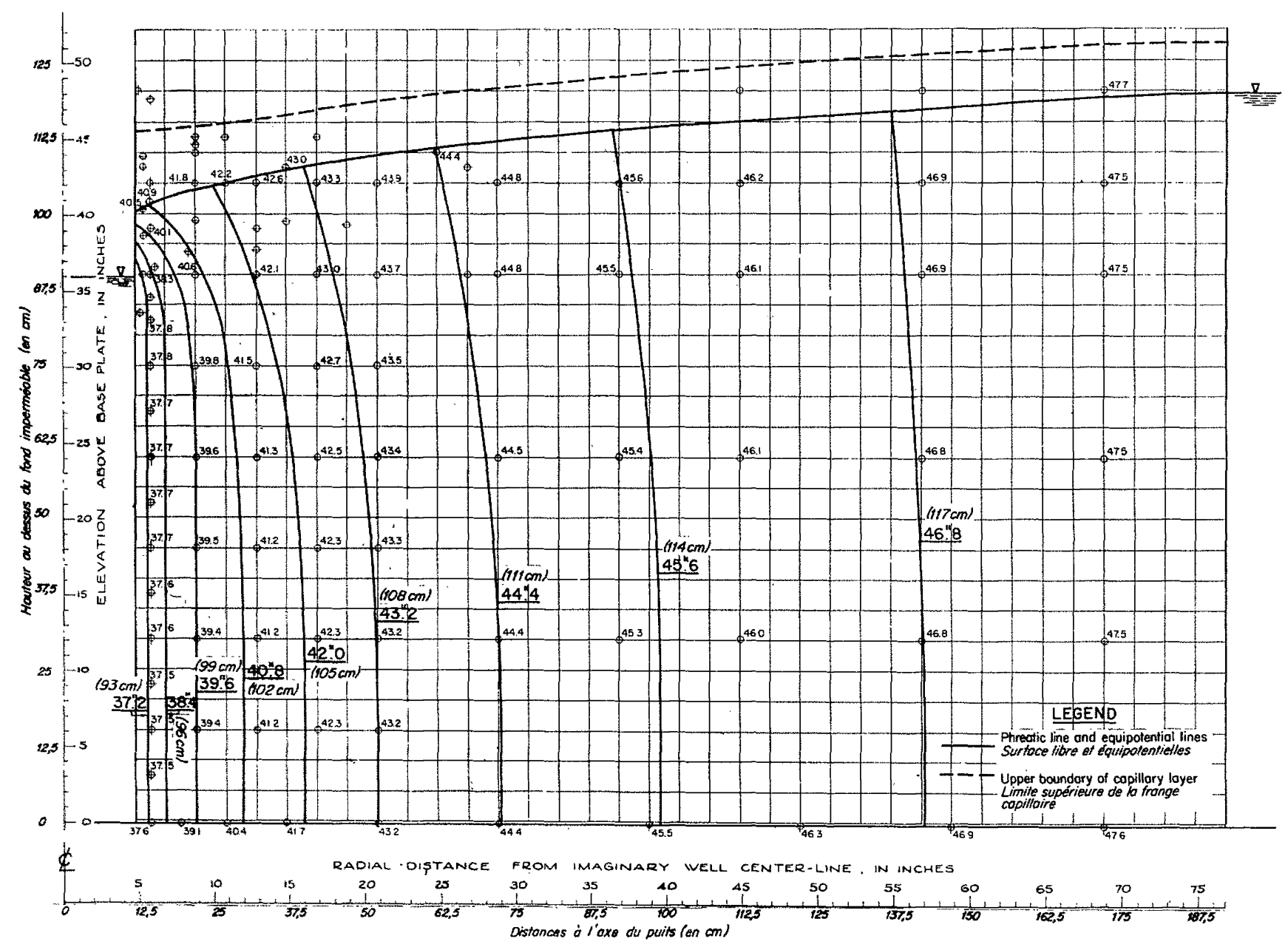

Fic. 12,- Series A, test 2 : Flow pattern from test data.

Série A, essai 2: Réseau d'écontement d'après les essais. 


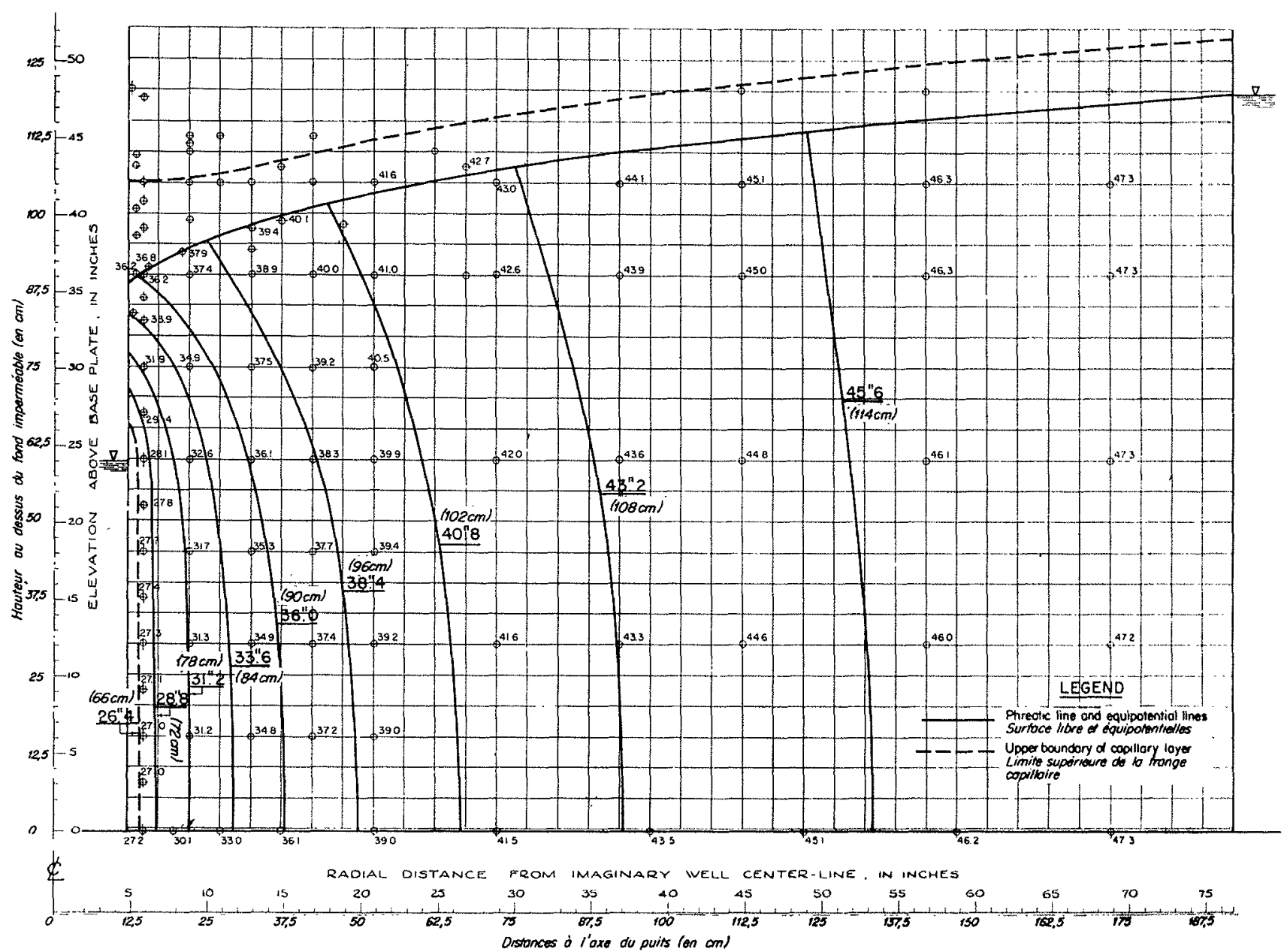

Fili. 13.--Series A, lest 4: Flow pallern lorom lesl data.

Serrie A, essai 't: Riseau d'aronlement d'apres les essais.

Jocation of the phrealic line occurred, which indicated that the disturbance was not enough to produce a serious distortion of the total flow pattern.

The failure of the piezometers to register any appreciable immediate change as Test 7 was finished and Test 8 was begun was the first evidence of serious air segregation. Under ideal conditions, reductions in piezomeler readings varying from negligible amounts near the entrance face to several inches at the intersection of base plate and discharge face would have been expected. However, with air voids already beginning to exert considerable resistance to flow, especially at the points of maximum anticipated change, it is concluded that the effect of general lowering of piezometric head was counteracted by the obstructive effect of the air voids.

Further indications of the effect of the local change in effective permeability appear in Figs. 10 and 11 . Fig. 10 was taken within several hours of the beginning of 'Test 8 . Fig. 11, with three additional flow lines introduced, was taken a day later, following the injection of bluing at all but the topmost of the eight starting points. Comparison of Figs. 10 and 11 indicates not only that the paths of the three later lines are not consistent with the original five, but also that the paths of the original five had shifted to varying degrees since the preceding day.

It was noted that llow lines which were drawn into the capillary layer ultimately returned to the region below the phreatic surface before reaching the discharge face. The paths of the two uppermost flow lines of Fig. 10 are plotted in Fig. 15 to illustrate this condition.

It should be noted that the phreatic line is not a flow line in a case where a capillary layer exists. Thus, the equipotential lines in Figs. 1215 do not necessarily intersect the phreatic line at right angles. The difference is usually small; but near the discharge face the deviation from a right angle is casily noticeable. 


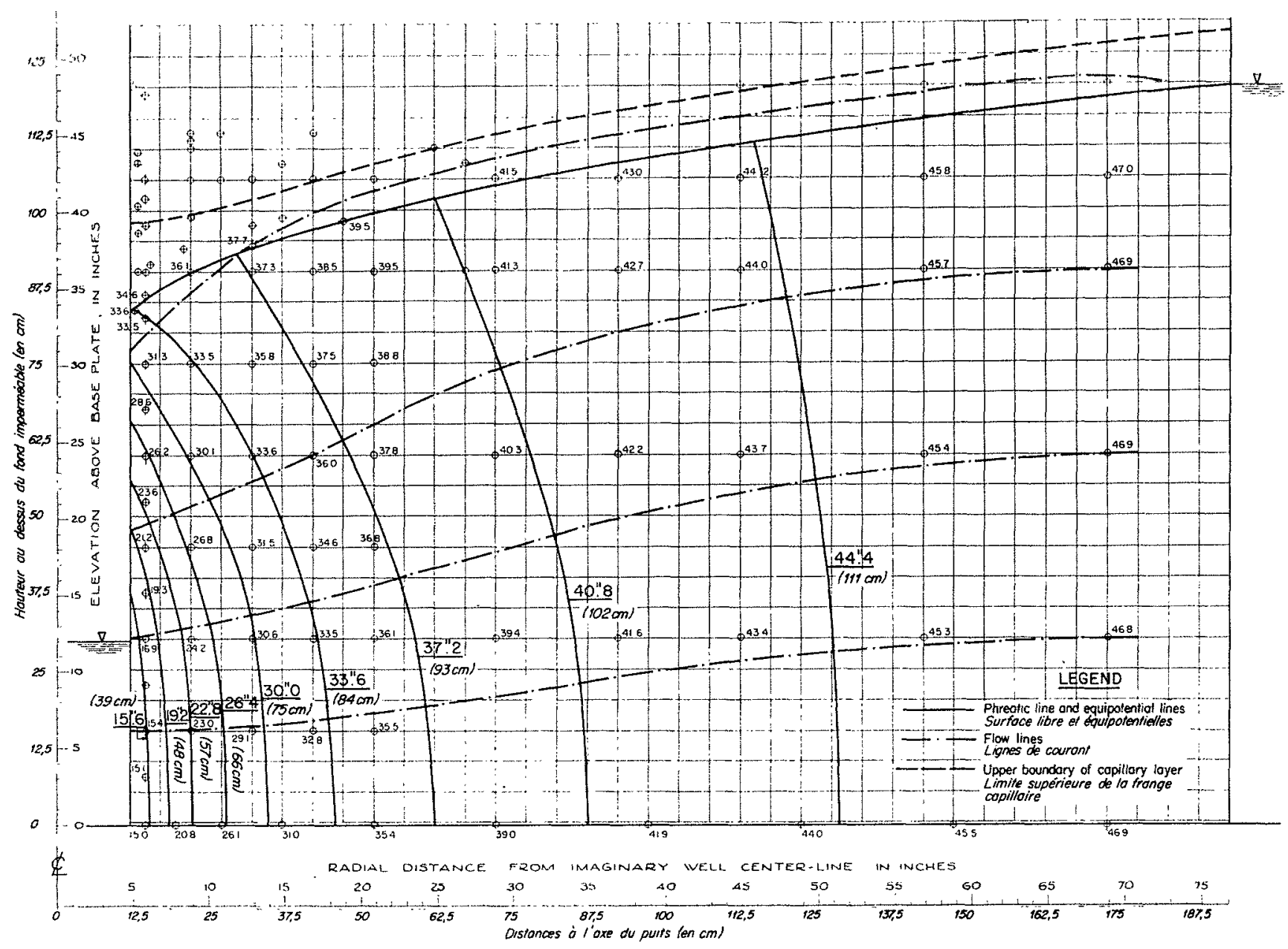

Fif. 14.-Series A, test $6:$ Flow pattern from test data.

Série A, essai 6 : Résean d'éconlement d'après les essais.

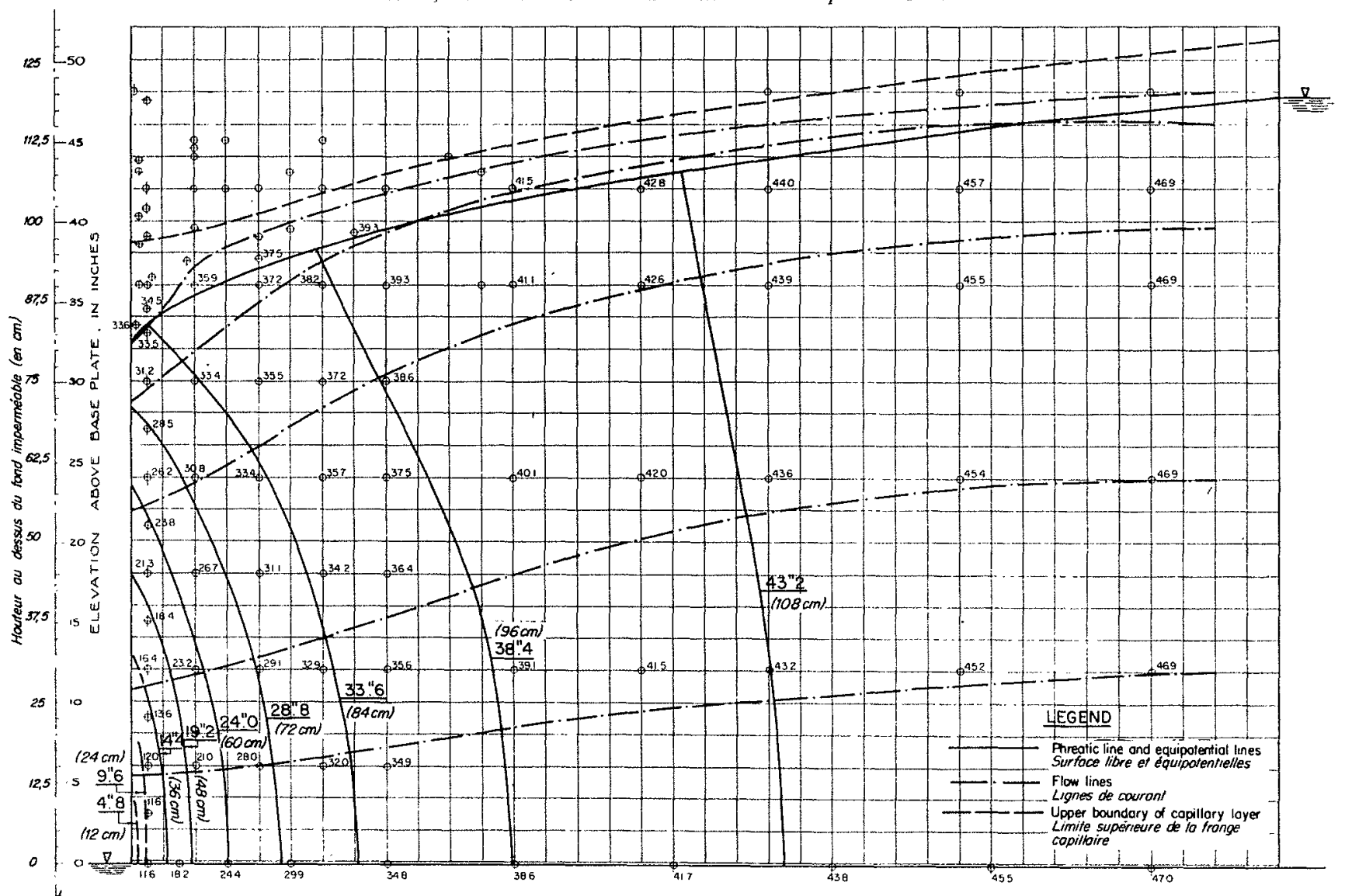

\& RADIAL DISTANCE FROM IMAGINARY IVELL CENTER-LINE IN INCHES

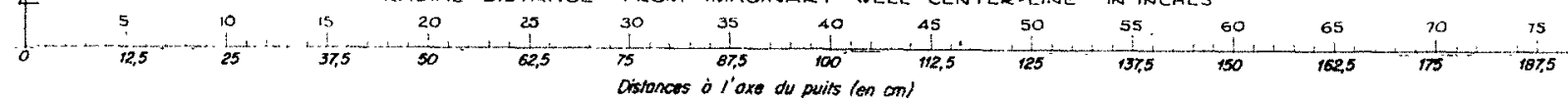

Fig. 15.-Series A, test $8:$ Flow pattern from test data.

Série A, essai 8: Réseau d'écoulement d'après les essais. 
3. Velocities.-The accuracy of the measurements recorded in Table 2 was insufficient to permil more than a qualitative appraisal of velocity relationships. 'Thus, the time required for the bluing to reach the discharge screen lrom the point of injection was least for the flow line nearest the hase plate, and greatest for the flow lines farthest away from the base plate.

Observation of the relative speed at which flow progressed through the capillary layer as compared to that at which it progressed through the region below the phreatic surface was of use in estimating the relative contribution of the capillary layer to the rate of flow.

4. Flow in the capillary layer.-A disproportionate difference may be noted in Table 2 in the times required for the uppermost pair of flow lines to reach discharge from their points of injection. Of this pair, the upper one, beginning at point 1 (see Key, Table 2), lay well within the capillary layer, while the other, beginning at point 2 , lay very close to the phreatic surface. Since point 2 was two inches below the water surface in the entrance-end standpipe, the relative amount of water diverted into the capillary layer was judged to be equivalent (o) that passing through the uppermost lwo inches of the entrance face. Assuming velocities to be about equal at entrance, the rate of llow through the capillary layer was estimated at about 4 per cent of total flow. Furthermore, throughout Series $A$, the consistent variation in total rate of discharge in proportion to the prediction of the Dupuit-Thiem equation indicated that the relative effect of the capillary layer remained essentially the same regardless of head differential.

On the basis of these observations, the relative increase in rate of flow as a result of the presence of a capillary layer was judged to be approximately equivalent to the ratio of half the thickness of the capillary layer to the tolal depth of water at entrance; that is :

$$
\frac{(\Delta Q)_{c}}{Q}=\frac{h_{c}}{2 \mathrm{H}}
$$

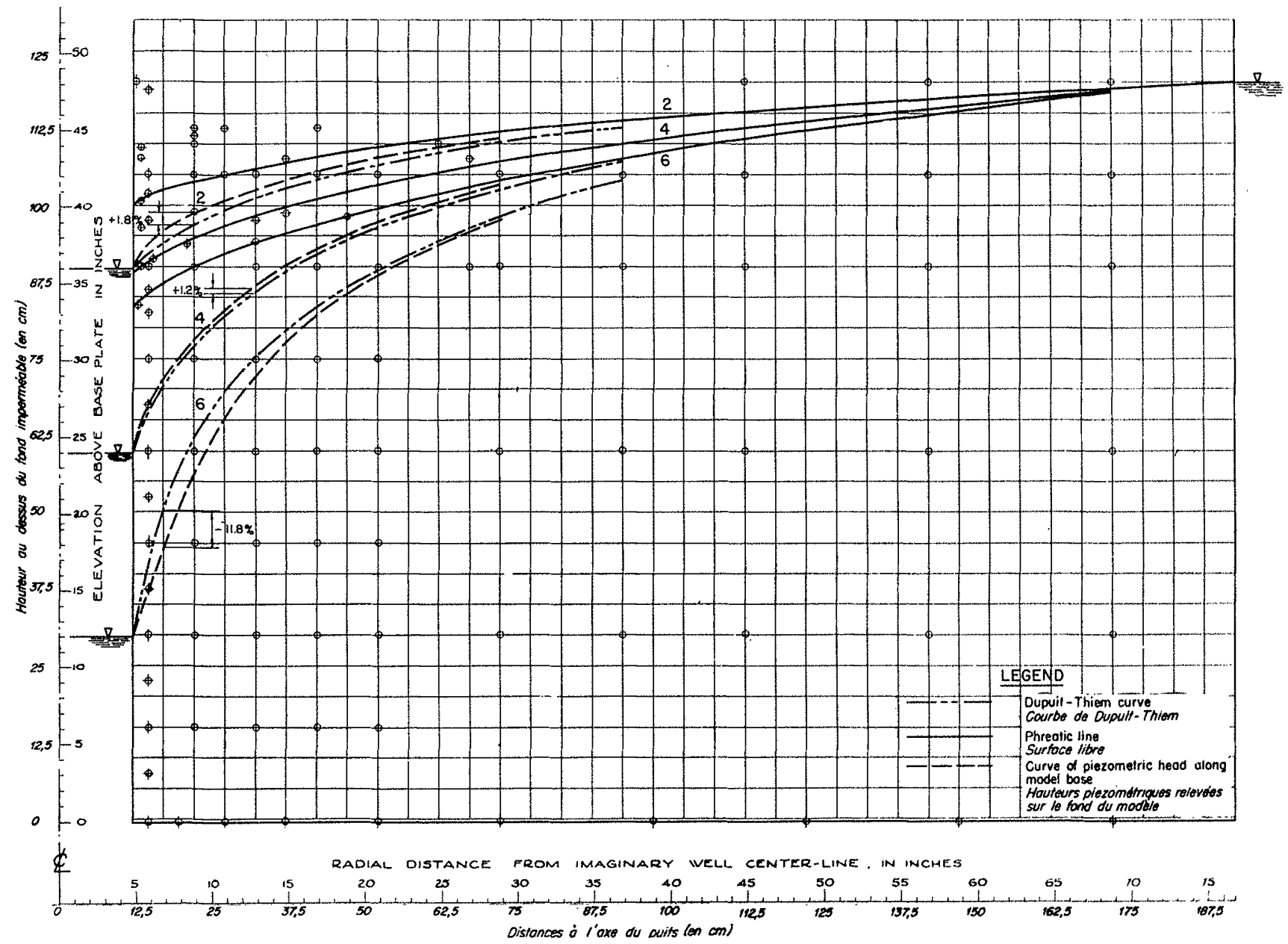

Fin. 16.-Series A, tests $2,4 \& 6$ : Comparison of Dupuit-Thiem curve and test curves.

Série A, essais 2, 4 et 6: Comparaison de la courbe de Dupuit-Thiem et des courbes expérimenlales. 


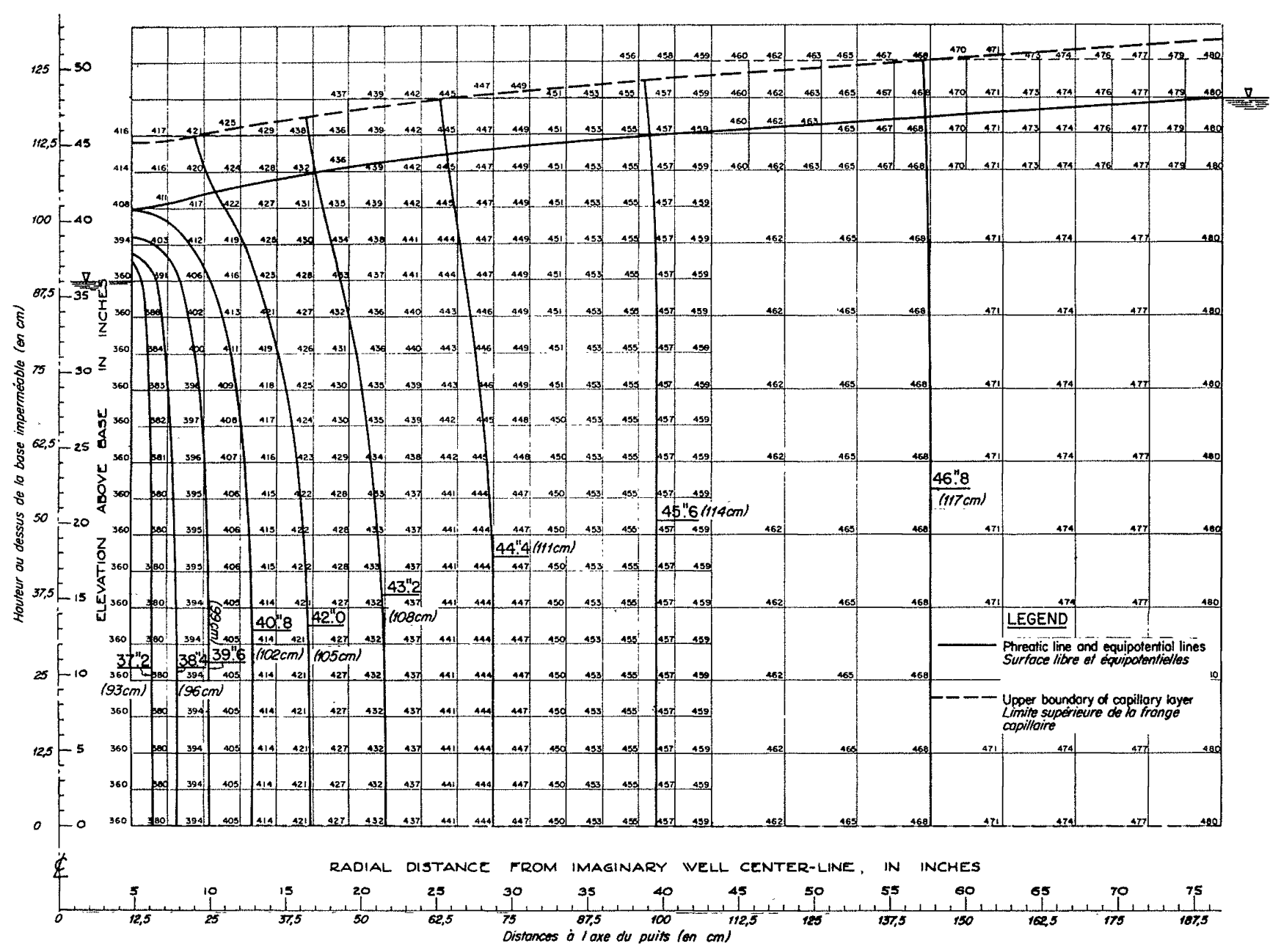

Fia. 17.-Series A, test 2 : Analysis by the relaxation method.

Série A, essai 2: Calcul par la méthode de relaxation.

where :

$(\Delta \mathrm{Q})_{e}=$ increase in rate of flow as a resull of the presence of a capillary layer.

$h_{c}=$ average thickness of capillary layer.

$\mathrm{H}=$ depth of water at entrance.

$Q=$ rate of discharge as determined from the Dupuit-Thiem equation.

5. Coefricient of permenillity.-Other investigations $[6,1]$ have shown that the average effective coefficient of permeability can be determined with salisfactory accuracy from the Dupuit-Thiem equation. The equation may be expressed in the form :

$$
k_{\mathrm{T}}=\frac{\mathrm{Q} \log _{e}\left(\frac{\mathrm{R}}{r_{0}}\right)}{\pi \mathrm{H}^{2}\left(1-\lambda^{2}\right)}
$$

where :

$k_{\mathrm{T}}=$ average effective coefficient of permeability at temperature $\mathrm{T}^{\circ}$ Centigrade, and :

$$
\lambda=\frac{h_{0}}{\mathrm{H}}
$$

However, the well model represents only one twenty-fourth of the region around a well; observed discharge rates should be corrected for the effect of the capillary layer; and the coefficient of permeability should be converted to that at $20^{\circ} \mathrm{C}$. Equation (2) then becomes:

$$
k_{20}=\frac{24 \mathrm{Q}_{\mathrm{T}}\left(1-h_{c} / 2 \mathrm{H}\right)}{\pi \mathrm{H}^{2}\left(1-\lambda^{2}\right)}\left[\log _{e}\left(\frac{\mathrm{R}}{r_{0}}\right)\right]\left(\frac{\mu_{\mathrm{T}}}{\mu_{20}}\right)
$$

where :

$$
\begin{aligned}
\mathrm{Q}_{\mathrm{T}}= & \text { observed rate of discharge from the } \\
& \text { model at temperature } \mathrm{T}^{\circ} \mathrm{C} \text {. }
\end{aligned}
$$

and :

$u_{r}, u_{20}=$ coefficients of viscosity of water at $\mathrm{T}$ and $20^{\circ} \mathrm{C}$ respectively. 
Table III contains the results obtained from the solution of equation (3) for the first seven lests of Series A. The average value of $k_{20}$ from these computations is 11.0 percent smaller than a value that was determined by a series of direct tests under similar conditions. It is concluded that the degree of saturation attained in the model in Series A was less than that obtained in the much smaller-scale direct permeability tests.

The consistency of the results recorded in Table 3 indicates that the effect of air segregation on the effective homogeneity of the sand was not serious during the first seven tests of Series A.

It was evident from the rapidity with which air segregation became an appreciable source of error during Test 8 that the opportunity for entrainment of air in the pipe line at discharge was a much more important factor in producing

\section{TABLE IIT.-COEFFICIENT OF PERMEABILITY} OF OTTAIVA STANDARD SAND

Tableau III. - Coefficient de PERMÉabilité DU SABLE TYPE D'OTTAWA

From direct permeability tests :

D'après les essais directs de perméabilité :

$k_{20}=0.460 \mathrm{~cm} / \mathrm{s}$ at (pour) $n 0.40$

From model tests data :

D'après le modéle:

$$
k_{20}=\frac{24 \mathrm{Q}_{\mathrm{T}}\left[1-\left(h_{c} / 2 \mathrm{H}\right)\right]}{\pi \mathrm{H}^{2}\left(1-\lambda^{2}\right)}\left(\ln \frac{\mathrm{R}}{r_{0}}\right)\left(\frac{\mu_{\mathrm{T}}}{\mu_{20}}\right)
$$

\begin{tabular}{|c|c|c|c|}
\hline $\begin{array}{c}\text { Series } \\
\text { Série }\end{array}$ & $\begin{array}{c}\text { Test } \\
\text { Essui n" }\end{array}$ & $\begin{array}{c}k=0 \\
(\mathrm{~cm} / \mathrm{s})\end{array}$ & $\begin{array}{c}\text { Ave ligo } \\
(\mathrm{moy} .) \\
(\mathrm{cm} / \mathrm{s})\end{array}$ \\
\hline \multirow{8}{*}{ A } & 1 & 0.410 & \multirow{8}{*}{0.409} \\
\hline & 2 & 0.412 & \\
\hline & 3 & 0.412 & \\
\hline & 4 & 0.408 & \\
\hline & 5 & 0.406 & \\
\hline & 6 & 0.405 & \\
\hline & 7 & 0.409 & \\
\hline & 8 & & \\
\hline \multirow{3}{*}{ B } & 1 & & \multirow{3}{*}{0.32} \\
\hline & 2 & 0.30 & \\
\hline & 3 & 0.35 & \\
\hline \multirow{3}{*}{ C } & 1 & 0.414 & \multirow{3}{*}{0.405} \\
\hline & 2 & 0.400 & \\
\hline & 3 & 0.402 & \\
\hline \multirow{3}{*}{ D } & 1 & 0.403 & \multirow{3}{*}{0.396} \\
\hline & 2 & 0.390 & \\
\hline & 3 & & \\
\hline
\end{tabular}

non-uniform llow conditions than the length of time during which the circulating water had been exposed to air. As indicated by the results of Table 3 , the constant exposure of the flowing water to air at the waler surfaces in the two standpipes as well as in the sand had nol caused appreciable inconsistencies over the four-week period taken for the series; but when the water level had been reduced to zero in the discharge standpipe, so that the discharge drainpipe (see Fig. 3) ceased to flow full, the development of air voids reached serious proportions within two days.

The effects of several of the important differences in test conditions among Series A, B, C, and $D$ are reflected in the values of average effective coefficient of permeability listed in Table 3. In Series B, during which no attempt was made to control air segregation, model test values of coefficient of permeability dropped an average of 22 percent below the value observed in Series A. In Series C and D, care was taken to reduce the amount of air segregation as much as possible without unloading and reloading the sand. The degree of saturation thus attained in the two series was judged to be about the same, so that comparable conditions of permeability were to be expected. The resulting observed values of coefficient of permeability differ only by 1 and 3 percent, respectively, from the Series A value.

It is concluded that the process of steady replacement of the water in the model by warmer water in small quantities over an extended period during continuous flow is an effective method of holding air segregation within limits sufficient to avoid serious variation of the value of the effective coefficient of permeability.

6. Phreatic Lines.-Study of the phreatic lines for the eight tests of Series A shows the decreasing effect of successive 6 -inch drops in the water level at discharge.

The data recorded in Table 1 for Series B show that the phreatic lines were higher for that group of tests than for the corresponding tests of Series A, although the effect of the capillary layer was smaller because its depth was the active, rather than the passive, value. The discrepancy can be explained by the non-uniform flow conditions. Piezometer readings and discharge rate varied throughout the duration of the tests. Air segregation, increasing in intensity toward the phreatic surface, could be observed through the side-walls over their entire length at all times. The effect was that of a nonhomogeneous porous medium. Since the extent and nature of the effective non-homogeneity could not be accurately gauged, no analysis of the data of Series B was attempted. 
The phreatic lines of Series D were least aflected by capillary action. In addition to the fact that the water had been chemically treated to reduce its surface tension to aboul 40 percent of its ordinary value, the degree of saturation within the capillary layer was undoubledly lower than was the case throughout most of the permeated part of the sand mass.

7. Comparison OF TEST curves With DUPUITTHIEM EQUATION.-Fig. 16 provides a comparison of three different curves for each of three tests of Series A. The three dot-dash curves are plots of the Dupuit-Thiem equation for Tests 2, 4 , and 6 ; the three dashed curves are plots of piezometric head along the base-plate, determined from the base-plate piezomeler readings; and the solid curves are the phrcatic lines, also determined from test observations. The Dupuit-'Thiem curve appears to be roughly accurate as an expression for the variation of piezometric head along the impervious base. The maximum discrepancy occurs in Test 6 and amounts to about 12 percent.

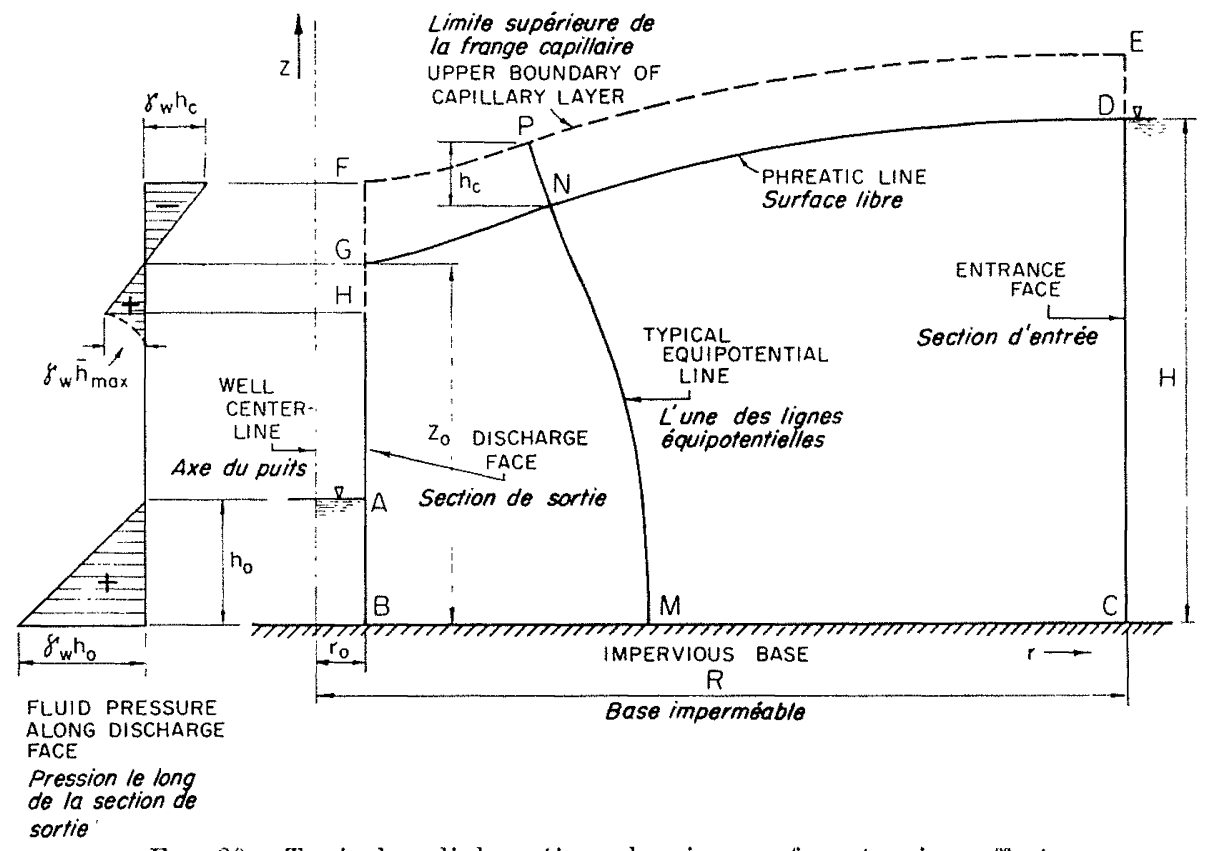

Fug. 20.-Typical radial section showing surface tension effects.

Coupe radiale montrant les effets de la tension superficielle.

\section{IV.-COMPARISON OF TEST RESULTS WITH RESULTS OF ANALYSIS BY RELAXATION METHOD}

\section{A) The Relaxation Method}

1. General.-The relaxation method is a method of successive approximations. It was originated by R. V. Southwell in England, and has been developed during the past twenty years by Southwell and a group of associates through application to a variety of problems in engineering and physics. For a detailed treatment of the method, reference is made to Southwell's texts $[4,5]$.

In applying the method to the analysis of the present group of problems, it has been found desirable to make use of a modification of the original procedure which is described briefly in the following paragraphs.
2. Application to SEEPAGE TOWARD A GRAVITX wELL. S. T. Yang [7] has adapted the relaxation method to the particular case of axially-symmetrical three-dimensional flow which is typified by the problem of seepage toward a fullypenetrating gravity well in a pervious stratum overlying a horizontal impervious base. It is this modification of the Southwell method which has been applied throughout the theoretical part of the present investigation. A brief statement of the important differences between Yang's and Southwell's procedures follows :

1. Yang's analysis is based on the compulation of piezometric head, rather than pressure head (Southwell's choice), as the poten- 


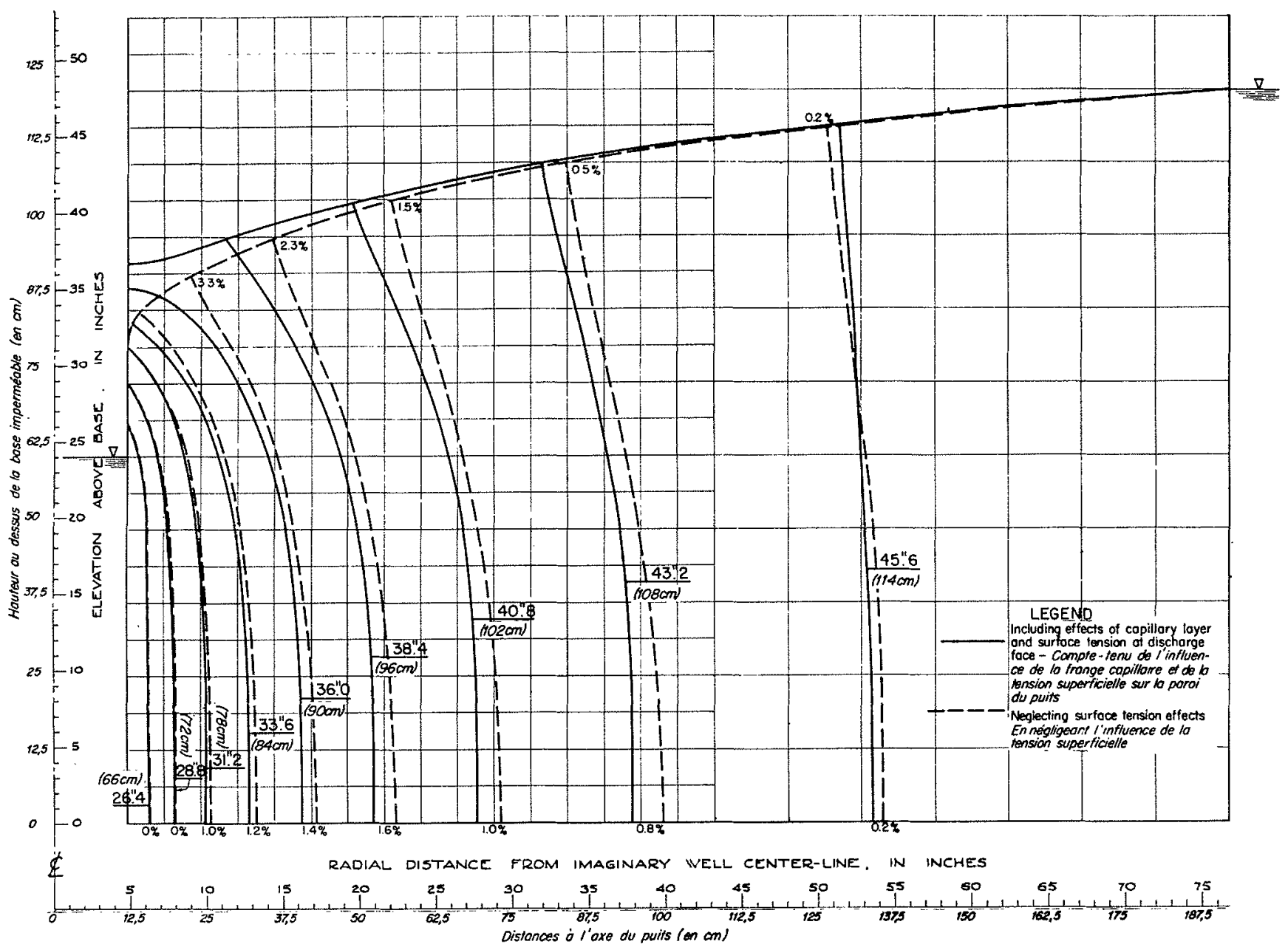

FIG. 21.-Series A, test 4 : Comparison of relaxation solutions based on different boundary conditions.

Série A, essai 4: Comparaison de diverses solutions obtenue par la méthode de relaxation en adoptant des conditions aux limites différentes.

tial function whose values at grid points are the immediate objective of the relaxation procedure.

2. Whereas Southwell, in a problem involving an initially unknown boundary (such as the phreatic line in the present case), fits the relaxation grid exactly to an estimate of the unknown boundary by the use of irregular stars $\left(^{*}\right)$ at nodes adjacent to that boundary, Yang approximates the estimated phreatic line with a grid whose strings and meshes are intact. Thus, for example, in the case of the square-mesh grid of Figs. 17-19, the upper boundary of the grid is a broken line composed of an irregular sequence of horizontal and ver-

(*) An irregular star is a group of strings, not all of which are of equal length, joined at a particular node adjacent to an irregular boundary. The strings which are not of standard length are those which join the node to the boundary. tical mesh sides which follows the curve of the phreatic line. The horizontal parts of the upper grid boundary are shown as dashed lines to indicate that they are assumed to be half-strings. A half-string is one which replaces one-half of a typical (full) string's share of potential surface, where the replaced area lies on but one side of the half-string. Thus, half-strings are correctly used along the base plate, where they are indicated in all the relaxation solutions (Figs. 17-19). But at the upper grid boundary, half-strings must be recognized as an approximation of the replaced potential surface.

Having introduced these changes in the process of setting up his gravity well problem, Yang applied the relaxation technique as developed by Southwell without further modification.

3. Discussion. - Yang's modification of the relaxation method was applied to the analysis 


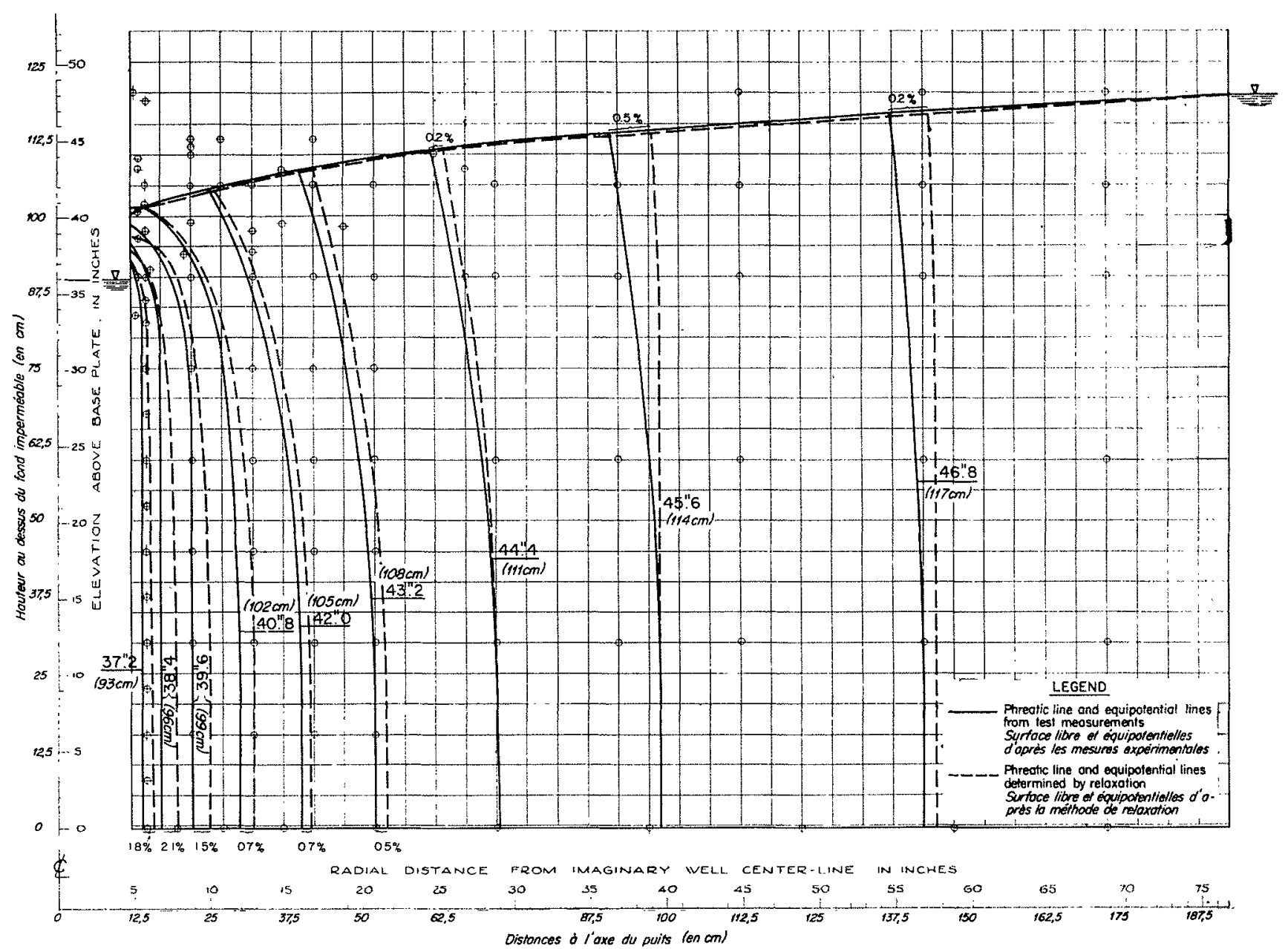

Fig. 22.--Series A, test 2 : Comparison of test curves and curves obtained by the relaxation method. Série A, essai 2: Comparaison des lignes d'ean expérimentales el des lignes d'ean oblenues par la méthode de relaxation.

of the problems of interest in the present investigation.

Two separate preliminary analyses of the case of Test 8 of Series A were carried through as familiarization exercises. The initial estimate in one case placed the phreatic line too low; in the other, too high. Upon completion of the relaxation process, it was found that the two solutions were not the same: The phreatic line corresponding to the higher initial estimate reached the discharge face at an elevation about 10 percent higher than the other above the impervious base. When the phreatic lines and equipotential lines were plotted from the final nodal values, it was found that the intersections were not at right angles in the case corresponding to the low initial estimate. Since the intersections in the other case satisfied this requirement, the solution arrived at from the higher initial estimate was judged the more accurate.

It is significant that careful application of the method, beginning with two different initial es- timates, had not yielded identical solutions-in spite of the fact that in each case convergence had been rapid at the outset. It is concluded that the difficulty lies in the nature of the approximation involved in the use of half-strings along the initially unknown grid boundary. Since the phreatic line is not horizontal, the horizontal halfstrings introduce an error which increases in importance as the slope of the line they are approximating increases; that is, the error increases in the direction in which more, rather than less, accuracy is essential.

4. Conclusion.- In the use of Yang's modification of the Southwell procedure, the automatic feature of the process of successive corrections is to some extent lost, so that the test of the correctness of a solution must include a review of the boundary conditions in addition to the reduction of residual forces to negligible amounts. It was found that the angle of intersection between equipotential lines and the phreatic line 
provided the decisive criterion upon which to base judgment of a solution.

\section{B) Analyses of Representative Test Cases}

1. Presentation.-Figs 17-19 show the relaxation solutions of Tests 2,4 , and 6 , respectively, of Series A.

The grid represents a radial section of the well model, with the well center-line indicated at the left. Elevations above the model base are indicated on the well center-line; and below the grid is a scale of radial distances, also in inches, measured from the well center-line. Water levels at entrance and discharge are shown in each figure. The phreatic line and nine equipotential lines dividing the region of flow into ten equal potential intervals are shown as heavy solid lines. The upper boundary of the capillary layer is represented by a heavy dashed line. The strings of the relaxation grid are represented as lighter solid (full strings) and dashed (half- strings) lines. The value of piezometric head, expressed in inches of water, corresponding to each equipotential line is noted in large figures beside the line; and the final nodal values of piezometric head are recorded in small figures at the upper left of each node. The unit in the case of all nodal values is one tenth of an inch of water-the limit of accuracy of piezometer readings. For convenience in numerical work, the length of side of the largest grid square was taken equal to the well radius; and the unit of radial distance, for purposes of the relaxation process only, was taken as one-half the length of side of the smallest grid square appearing in the solution $\left(^{*}\right)$.

2. BOUNDARY CONDITIONS.-Fig. 20 is a sletch of a typical radial section similar to those represented by Figs. 17-19.

Line CDE represents the entrance face; line

(*) These radial distances have no significance beyond their part in the liquidation of residuals.

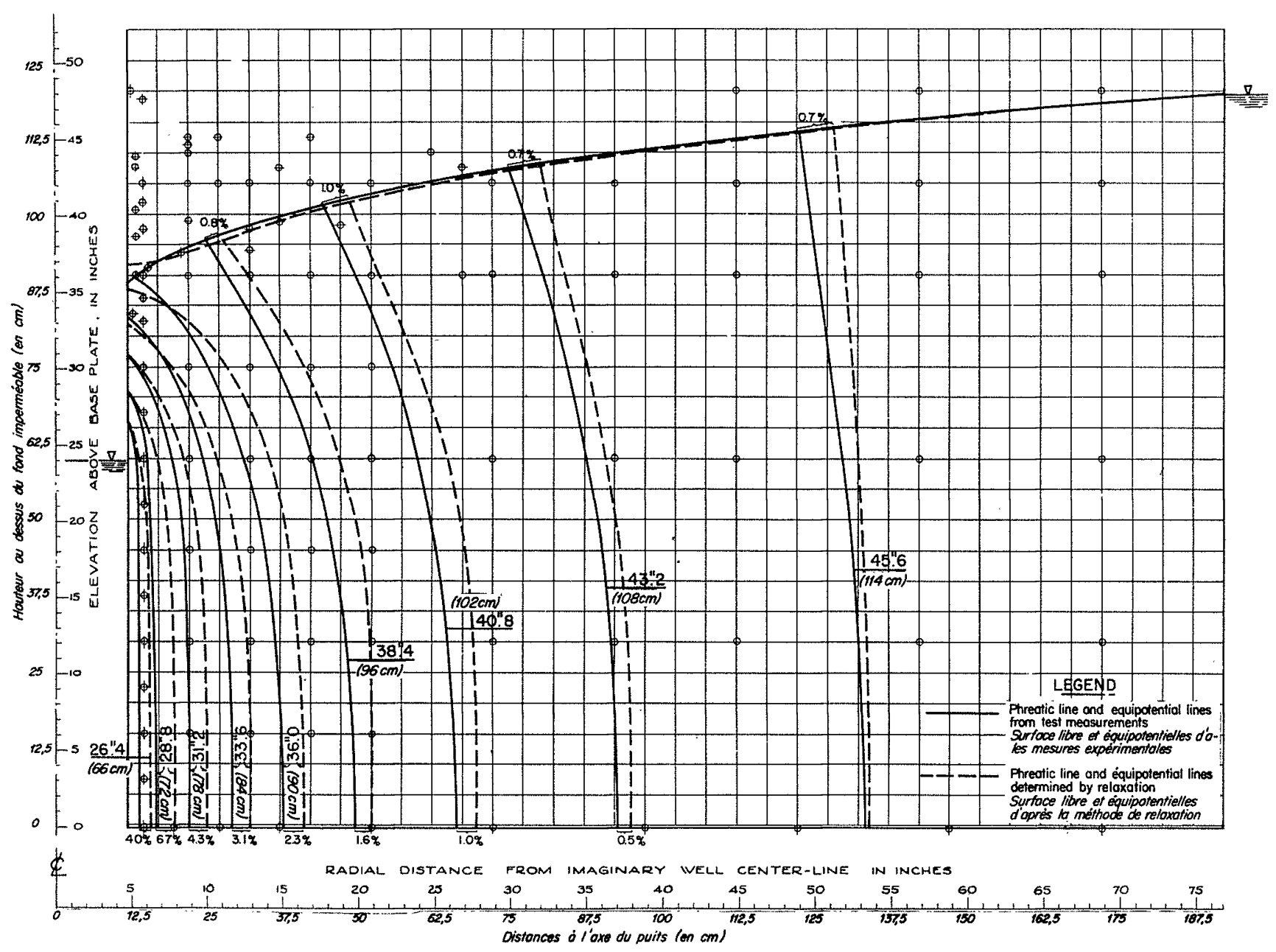

FIi. 23,-Series A, test 4: Comparison of test curves and curves obtained by the relaxation method. Série A, essai 't: Comparaison des lignes d'eau expérimentales et des lignes d'eau obtenues par la méthode de relaxation. 


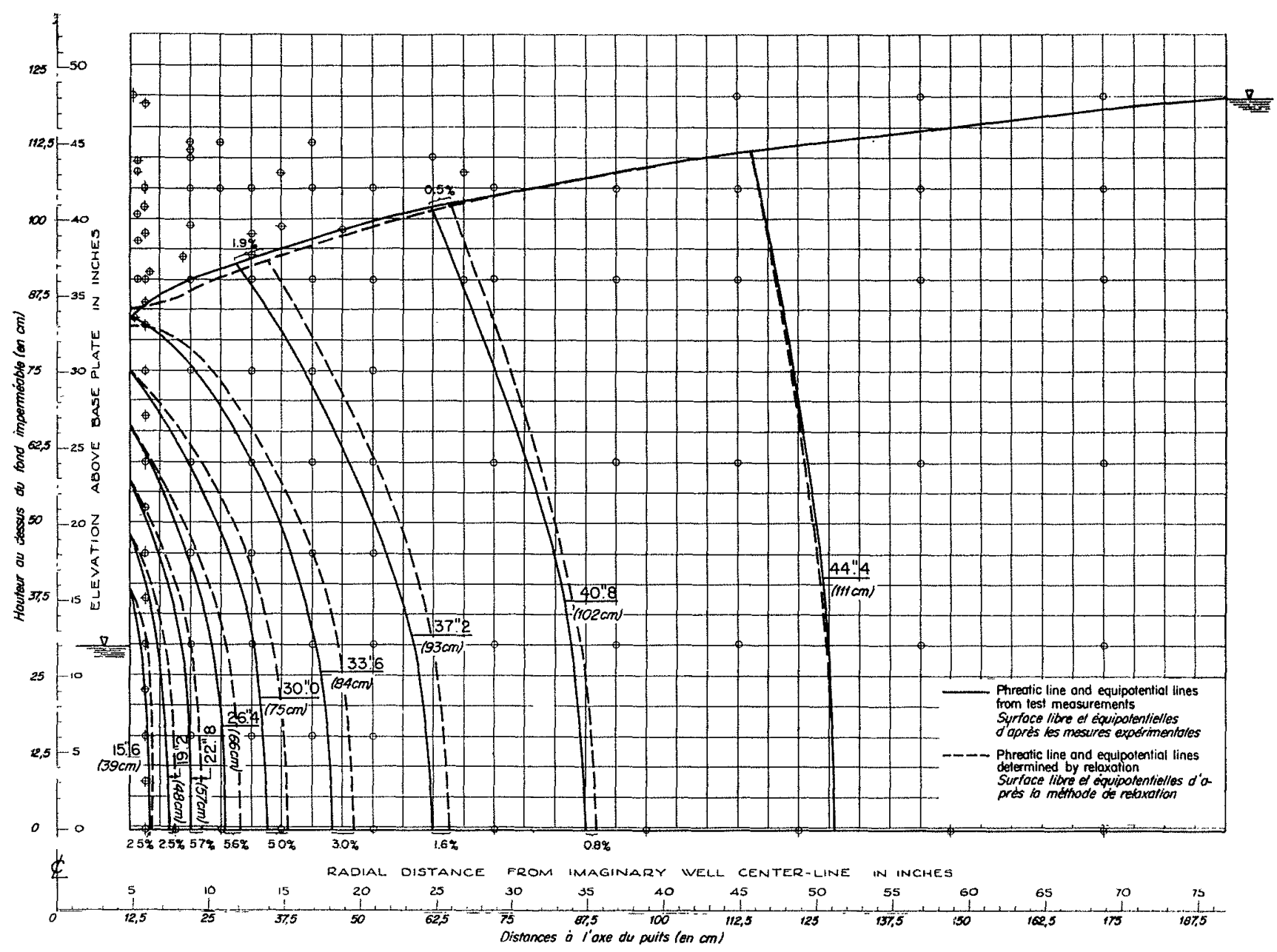

Fri. 24.--Series A, test 6: Comparison of test curves and curves obtained by the relaxalion method. Série A, essai 6: Comparaison des lignes d'eau expérimentales et des lignes d'eru obtennes par la méthode de relaxalion.

BAHGF, the discharge face. Line BC corresponds to the model base. The water surface at entrance is indicated at $\mathrm{D}$ at an elevation $\mathrm{H}$ above the base; at discharge, at $\mathrm{A}$ at an elevation $h_{0}$ above the base. Line DNG represents the phreatic line; line $\mathrm{EPF}$, the upper boundary of the region of seepage. Axes of coordinates $r$ (radial distance) and $z$ (elevation) are taken as the impervious base and the well center-line, respectively; and the elevation of the point of intersection of the phreatic line and the discharge face is designated as $z_{0}$.

The boundary conditions for the case shown, in the form in which they were applied to the analysis of Tests 2, 4, and 6 of Series A, may be expressed as follows :

1. From A to B :

$$
\varphi=h_{0}
$$

where $\varphi=$ piezometric head; that is, the line $A B$ is the final equipotential line in the direction of flow.
2. From B to $\mathrm{C}$ :

$$
\frac{\partial \varphi}{\partial z}=0
$$

that is, the line $B C$ is a flow line, so that every equipotential line must intersect it at a right angle.

3. From C to D :

$$
\varphi=\mathrm{H}
$$

that is, the line $\mathrm{CD}$ is the first equipotential line in the direction of flow.

4. From D to $G$ (through $E, P$, and F) :

$$
\varphi=z-h_{0}
$$

and :

$$
\frac{\partial \varphi}{\partial n}=0
$$


where $h_{c}=$ height of capillary rise and $n=$ direction normal to the boundary at any point.

The first of these two conditions follows from the fact that the phreatic line, DNG, must satisfy the condition : $\varphi=z$, so that the piezometric head at a point on the upper boundary of the capillary layer must be less than its own elevation by the amount of capillary tension in the water. The second condition follows from the fact that the line DEPFG is a flow line.

\section{From G to $A$ :}

Since point $G$ is on the phreatic line, the piezometric head is equal to the elevation, $z_{0}$, so that the water pressure is zero at that point. However, no flow out of the sand mass will oceur for some distance below point $G$ because the surface tension of the fluid is capable of producing back pressure against the tendency of the fluid to seep through the screen. When the water pressure becomes large enough to overcome the back pressure (say at point $H$ ), flow out of the sand mass will occur at that elevation.

A sketch of the distribution of water pressure along the vertical face $F G H A B$ is shown in the figure. Point $F$, where a right angle in the flow line occurs, is a dead corner. Thus a maximum amount of tension acts at this point. Procecding downward, the tension decreases, becomes pressure at some point, $G$, and finally reaches a value, say $\bar{h}_{\max }$, which is sufficient to overcome the resistance to lateral flow due to surface tension at the discharge screen. The rate of change of fluid pressure along FGH is less than that corresponding to hydrostatic distribution because some of the total potential contributes to maintenance of flow along the flow line FGH. Thus, the distance, FGH, is greater than the sum, $\left(h_{\mathrm{e}}+\bar{h}_{\max }\right)$. Also, $\bar{h}_{\max }$ is much less than $h_{c}$.

The remainder of the boundary (line HA) constitutes the discharge surface. However, since the fluid pressure at $H$ is greater than zero, the simple relationship, $0=z$, does not hold over the entire distance, HA. It seems reasonable to assume that the excess pressure is dissipated a short distance below point $H$, so that the greater part of the line HA docs satisfy that relationship.

The boundary conditions from $G$ to A may therefore be summarized as follows :

From $\mathrm{G}$ to $\mathrm{H}$ :

$$
\frac{\partial \varphi}{\partial r}=0
$$

since this much of the line is a flow line.

From $\mathrm{H}$ to $\mathrm{A}$ :

$$
\varphi=z+h^{\prime}
$$

where :

$$
\bar{h}_{\max } \geqq h^{\prime} \geqq 0
$$

that is, line $\mathrm{HA}$ is neither a flow line nor an equipotential line; nor can it properly be described as a free discharge surface except over as much of it as is unaffected by the excess fluid pressure whose maximum value occurs at point $H$.

3. Procedure.-The following numerical values were used in all three solutions under consideration :

and :

$$
h_{c}=3.6 \mathrm{in} .
$$

$$
\bar{h}_{\max }=1.0 \mathrm{in} \text {. }
$$

The value of height of capillary rise, $h_{c}$, was obtained by measurement, in Figs. 12-14, of the vertical projection of the distance along an equipotential line from the upper boundary of the capillary zone to the phreatic line.

The value of $\bar{h}_{\max }$ was estimated on the basis of some auxiliary tests on smaller-scale twodimensional seepage models consisting of sand placed between wooden plates about one inch apart.

Arbitrary location of point $H$ was necessary in order to begin the relaxation process. The relaxation process was then used as a basis upon which to judge the reasonableness of the original location of point $\mathrm{H}$, as well as the value of $\bar{h}_{\max }$, since accuracy in either estimate could be obtained only by a trial-and-error procedure.

4. Discussion.--In the following discussion, Fig. 20 is identified with Figs. 17-19 so that points, distances, and heads noted in Fig. 20 are used without further identification to designate their counterparts in Figs. 17-19.

The most important feature of the relaxation solutions of Tests 2, 4, and 6 of Series $A$ is the character of the flow pattern in the upper part of the region of flow at the discharge end. Although line DEPFGH is a flow line, it will be noted that its slope becomes zero as it approaches point $F$, and that it changes direction at a right angle at that point. The point $F$ is therefore a dead corner.

The back pressure effect appears, in Figs. 1719 , to distort the phreatic line in a manner similar to its effect on the boundary flow line. Note, however, that under the boundary conditions assumed in these analyses, the phreatic line is not a flow line, just as it is not a flow line in the model.

Fig. 21 is a plot of two flow patterns deter- 


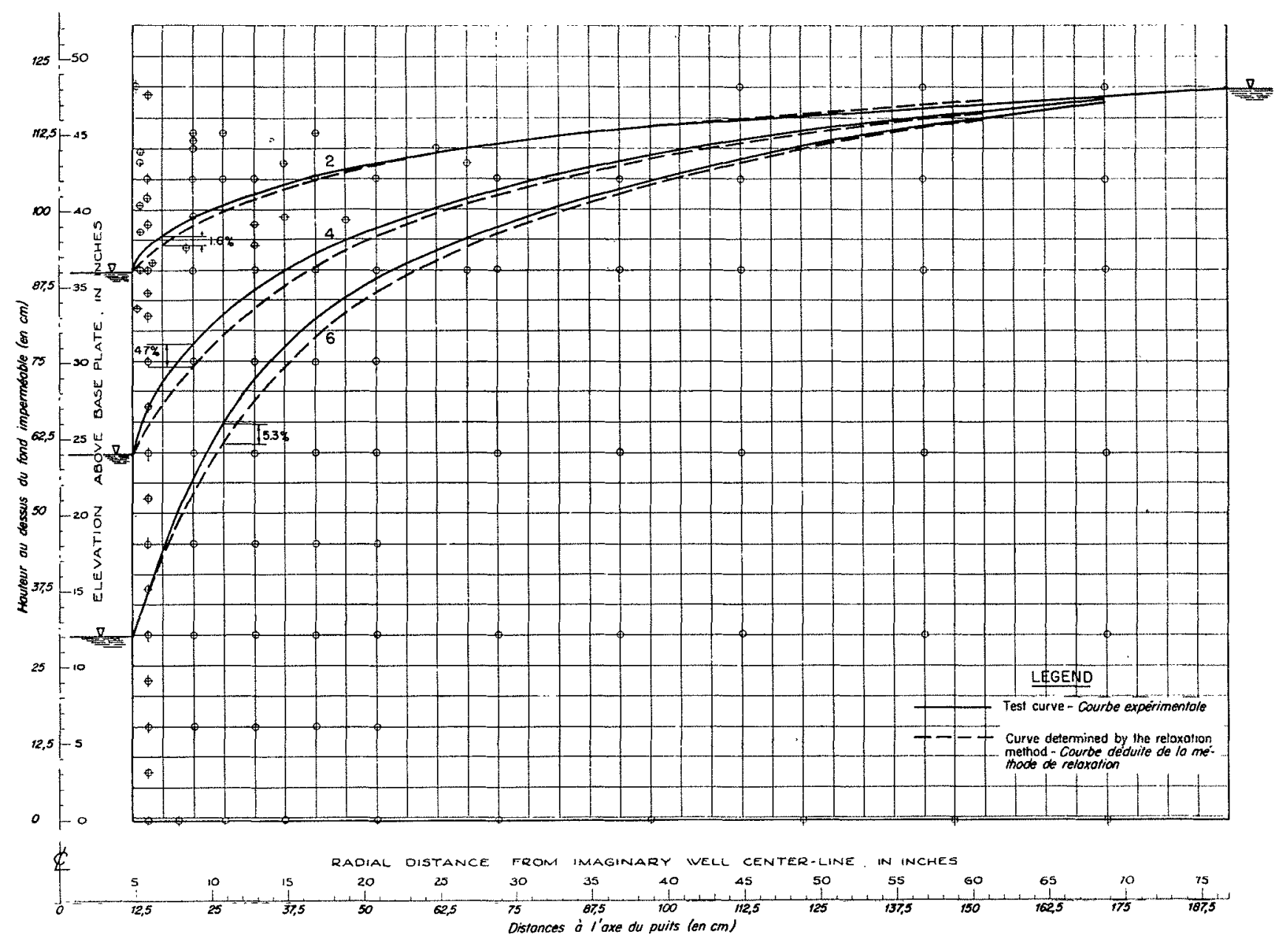

Fic, 25.--series A, tests 2,4 \& $6:$ Base piezometric head : comparison of test curres and curves determined by the relaxation method.

Série A, essais 2, 4 el 6 : Hauleur piézométrique sur le fond (comparaison des résullals experimenlunx et de ceux obtenus par la milhode de relaxution).

mined by the relaxalion method for a case corresponding to Test 4 of Series A. The solid lines show the flow pattern of Fig. 18 while the dashed lines indicate the flow pattern if the capillary layer and back pressure effects are neglected. It can be seen that the distortion caused by the capillary layer and back pressure effects is confined to a relatively small area in the upper part of the region of flow at the discharge end.

The values of $h_{c}$ am $\bar{h}_{\max }$ were relatively small in the present group of solutions because of the coarseness of the sand. The corresponding effect in the case of a finer material, and therefore of most natural materials, would be greater.

The location of point $\mathrm{H}$ (Fig. 20) in a particular solution must follow a cut-and-try procedure. Once the point has been selected and the appropriate value of piezometric head $\left(\varphi=z+h_{\operatorname{mix}}\right)$ assigned to it, a solution can be obtained by the relaxation process. The correct- ness of this solution could be checked by direct observation of the relative values of $\mathrm{GH}$ and $\mathrm{HA}$; but the construction of the model made this impossible, so the original location of $\mathrm{H}$ in the analyses of Tests 2, 4, and 6 of Series A was based on the results of auxiliary tests, and minor adjustments were made during the relaxation procedure.

\section{C) Comparison of Test Results with Relaxation Solutions}

1. Presentation.-In Figs. 22-24 the relaxation solutions of Tests 2, 4, and 6 of Series A are superimposed upon the corresponding plots of test results. The upper boundary of the capillary layer is not included in the comparison, since the correspondence between test and theory for this line follows very closely that for the phreatic line in all cases.

The deviation of each theoretical equipotential line from the corresponding test curve is 
expressed as a percentage of the theoretical potential value at the upper or lower extremity of the test curve. In nearly every case the value thus noted represents the maximum discrepancy between test and theory for that equipotential line.

2. Phreatic Lines.--Very close correspondence between test and theory will be noted for all three phreatic lines.

The maximum discrepancy in each case occurs at the point of intersection of the phreatic line and the discharge face. At this point the theoretical curve bends somewhat sharply back to a nearly horizontal slope, while the test curve does not show this final break. The difference can probably be explained in part by the limited number of piezometer readings which were obtained within the last two inches, where the deviation occurs. The test phreatic lines could have been drawn with a small reverse curve at the end without conflicting with the piezometer readings; but such an interpretation could not be justified on the basis of test data alone.

3. Equipotentral Lines.-Figs. 22-24 indicate that the largest discrepancies between corresponding observed and theoretical equipotential lines occur within the last three or four potential intervals before reaching the discharge face, and that the maximum deviation occurs from two to four potential intervals back from the discharge face.

Since separation of air out of solution was the major cause of differences between observed and theoretical flow patterns, it would be expected that the greatest differences would occur where fluid pressure was lowest, or where the gradient was the highest. However, the lowest fluid pressures occurred along the phreatic line and discharge surface; and the highest gradients occurred at the intersection of the free discharge surface and the surface of the water in the well. Consequently, it is evident that the regions of lowest fluid pressures and highest gradients do not coincide entirely with the regions of maximum differences between observed and theoretical flow patterns. It is concluded that the basis of computation of per cent errors may be somewhat misleading. For example, if the differences between observed and theoretical piezometric heads were compared to $h_{0}$, which is the minimum in any case, rather than to zero, the region of greatest per cent deviation would shift toward the discharge face. Or if the per cent errors were computed by comparing the observed and theoretical gradients, the shift would be considerably more marked in the same direction. However, the deviations computed by comparing gradients would be of questionable value except on a relative basis because the observed equipotential lines are in all cases smooth curves drawn as nearly as possible through series of points interpolated from observed piezometric heads.

4. Base PIEzometric HeAD.-Fig. 25 compares the curves of base piezometric head determined from the relaxation analyses with those plotted from test measurements. Per cent deviations between observed and theoretical curves are noted on each pair of curves. The values are taken at points of maximum difference.

Fig. 25 shows that the relation between observed and theoretical base piezometric head is characteristic of the general behavior of the complete flow pattern: Deviation of observed from theoretical values increases from test to test; and the maximum deviations noted are in the neighborhood of 5 per cent.

Comparison of Figs. 17 and 25 indicates that the relaxation method provides curves of base piezometric head which are in closer agreement than the Dupuit-Thiem curve with test observations.

\section{V. - SUMMARY AND CONCLUSIONS (*)}

\section{A) The Relaxation Method}

Three representative cases were selected from among the group of model tests performed in

$\left(^{*}\right)$ A comparison of the results of this investigation with those of Babbitt and Caldwell, Muskat, Yang, and others reported before 1951 has been largely omitted from the present condensation. However, since references to these investigators do appear, it is perhaps fitting, for the sake of completeriess, to add that several investigations of more recent date of publication have treated the same problem. Of these, two that have come particularly close to the present study in their objectives and scope are those of Boulton [8] and Hansen [9]. this investigation, and analyzed by means of the relaxation method.

The analyses differed from earlier analyses of similar problems in that the effect of the surface tension of the fluid upon the flow pattern was taken into account in a manner which is believed to be qualitatively accurate if quantitatively only approximate. The surface tension of the fluid is understood to be responsible for two relatively important features of the flow pattern, namely, the capillary layer, and the absence of discharge, because of back pressure against outward flow, through the discharge 


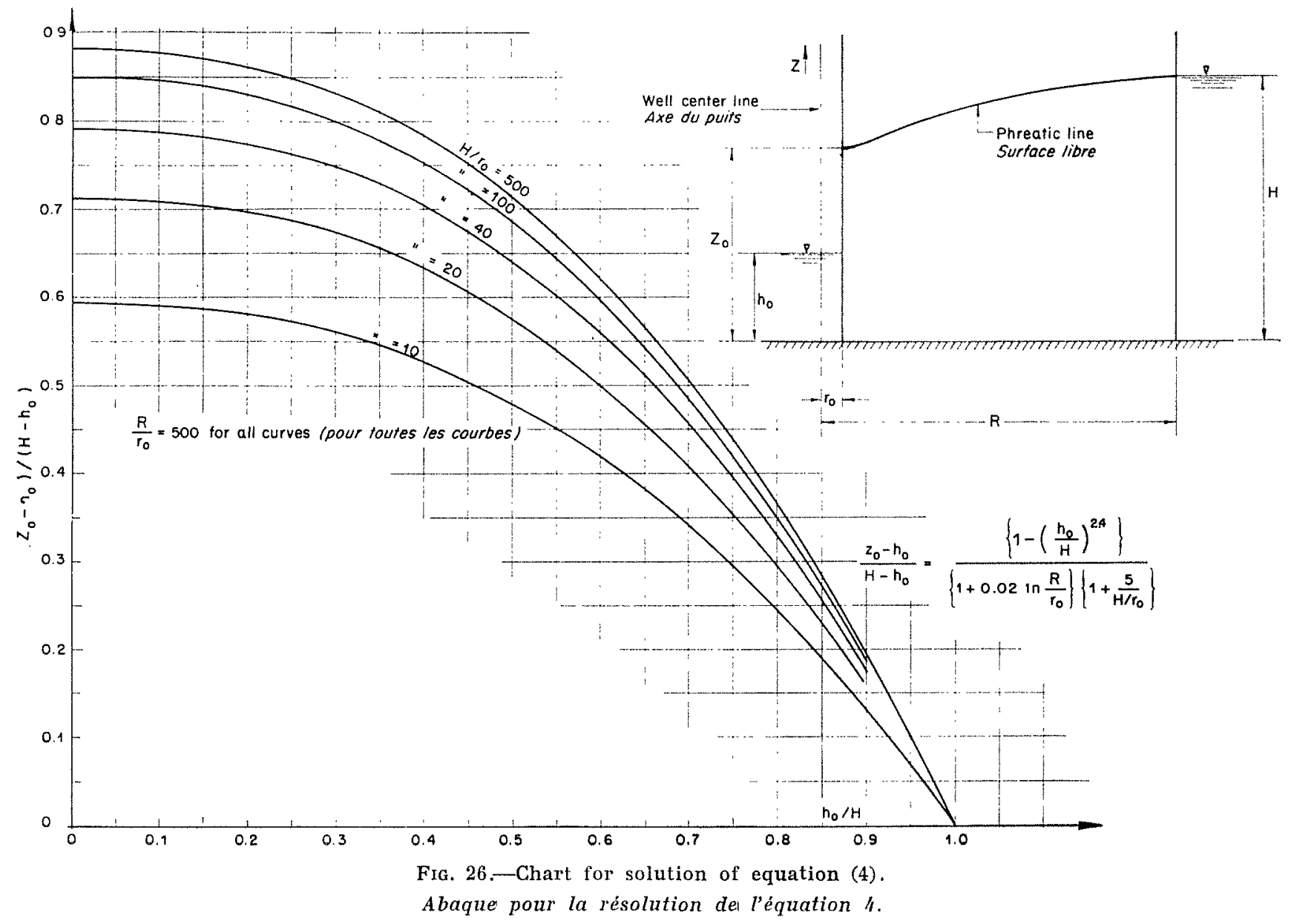

screen over a certain distance immediately below the phreatic surface. Evaluations of these effects were based on model-test observations and some auxiliary tests.

Satisfactory agreement was obtained between test results and relaxation solutions. On the basis of this group of comparisons, it is judged that a maximum discrepancy of about 6 per cent between actual and computed piezometric heads may be expected in the relaxation analysis of problems involving seepage toward a gravity well.

It is concluded that the relaxation melhod is a reliable analytical procedure for the determination of the flow pattern around a gravity well. In addition, the analysis of seepage toward a well has demonstrated the flexibility of the technique in handling boundary conditions which would make the application of more formal methods extremely difficult.

\section{B) The Phreatic Line}

1. General.-The conventional theoretical conception of a phreatic line is that of a flow line which forms a well-defined boundary between the permeated and the non-permeated parts of a porous medium supporting gravity flow.

In the light of the relaxation analyses and the test observations of the present investigation, the phreatic line loses some of its significance. It is neither a boundary nor a flowline; it is simply a particular equi-pressure line. However, it is an important equi-pressure line, because the upper boundary of the capillary layer can be most conveniently expressed with reference to the phreatic line, and because for practical purposes the phreatic line may be considered the upper boundary of the region of flow.

The shape of the phreatic line at the discharge face has been shown to be somewhat different in detail from that usually represented. It is not a flow line; and because of the dead corner it does not meet the discharge face by curving tangent to it as would be the case in the absence of surface tension in the seeping fluid. Nor does it intersect equipotential lines at right angles.

Analysis has further indicated that the effect of surface tension is to raise the phreatic line over its entire course by an amount which reaches a maximum at the discharge face. 
2. Empirical equations.-Since it has been possible to obtain satisfactory agreement between observed and theoretical results, it would be of use to develop equations which locate and describe the phreatic line in terms of convenient characteristic ratios. Two empirical expressions are therefore proposed on the basis of the results of the present investigation. The first expresses the value of the vertical dimension of the free discharge surface. Referring as usual to a radial section, the proposed equation reads :

$$
\left(\frac{z_{0}-h_{0}}{\mathrm{H}-h_{0}}\right)=\frac{\left[1-\left(\frac{h_{0}}{\mathrm{H}}\right)^{2 . t^{-}}\right]}{\left[1+\frac{1}{50} \log _{e}\left(\frac{\mathrm{R}}{r_{0}}\right)\right]\left[1+\frac{5}{\left(\mathrm{H} / r_{0}\right)}\right]}
$$

where the symbols denote the distances so marked in Fig. 20.

The form of the right-hand side of equation (4) implies that the most important of the three characteristic ratios is $h_{0} / \mathrm{H}$, and that $\mathrm{H} / r_{0}$ and $\mathrm{R} / r_{0}$ follow in that order.

Fig. 26 is a chart for the solution of equation (4). Since the value of $\left(z_{0}-h_{0}\right) /\left(\mathrm{H}-h_{0}\right)$ is least sensitive to variations in $\mathrm{R} / r_{0}$, the value of the Iatter has been taken as 500 for all curves. The figure may be used directly to determine the value of the ratio, $\left(z_{0}-h / / \mathrm{H}-h_{0}\right)$ for any combination of characteristic ratios of which $\mathrm{R} / r_{0}=500$ is one; and for values of the ratio $\mathrm{R} / r_{0}$, other than 500 , a correction factor (usually insignificant) may be determined without difficulty from consideration of the appropriate term in equation (4).

Since equation (4) locates only the extremity of the phreatic line, the following equation is proposed to express its path :

$$
\frac{z-z_{0}}{\mathrm{H}-z_{0}}=2.5\left(\frac{r-r_{0}}{\mathrm{R}-r_{0}}\right)-1.5\left(\frac{r-r_{0}}{\mathrm{R}-r_{0}}\right)^{1.5}
$$

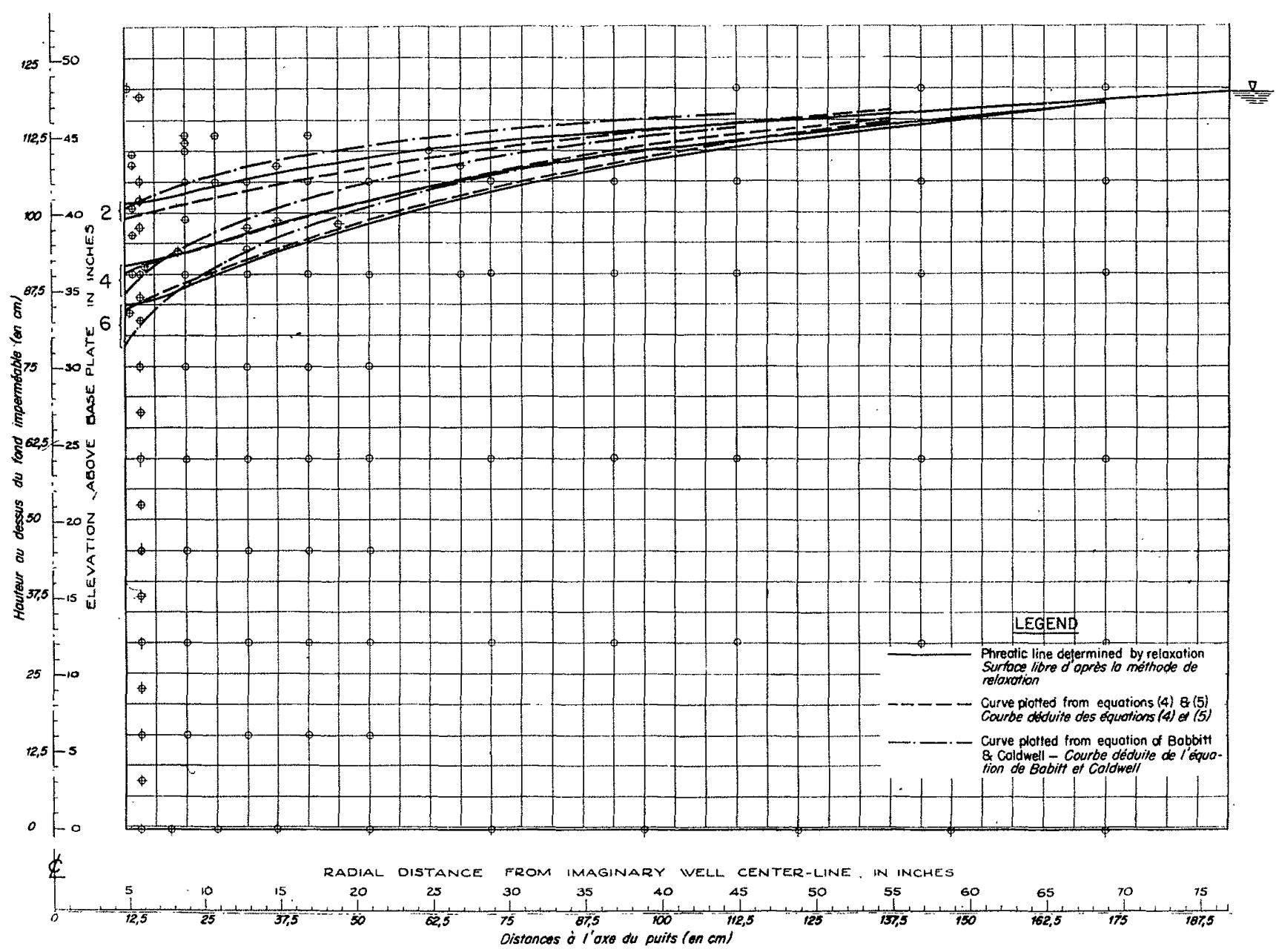

Fig. 27.-Series A, tests $2,4 \& 6$ : Comparison of phreatic lines determined by the relaxation method with curves plotted from empirical equations.

Série A, essais 2, 4 et 6 : Comparaison des surf́aces libres déterminées par la méthode de relaxation avec celles obtenues à partir des formules empiriques. 
A comparison of the curve of equation (5) with the theoretical phreatic lines for Tests 2,4 , and 6 of Series A appears in Fig. 27. Curves plotted from the equation proposed by Babbitt and Caldwell [1] for the phreatic line are also shown.

In applying equations (4) and (5) to a particular case, it is suggested that the coordinates of some point $(r, z)$ be taken from observations or from the Dupuit-Thiem curve at some distance, say $50 r_{0}$, from the well, where Dupuit's assumptions are valid, and substituted in the empirical equations for the point $(\mathbf{R}, \mathbf{H})$. Thus, the phreatic line of equations (4) and (5) will constitute an end segment to be attached at the discharge end of a phreatic line whose remainder can be determined with acceptable accuracy by the Dupuit-Thiem equation.

\section{C) The Capillary Layer}

The effect of the capillary layer on the position of the phreatic line has been discussed in the preceding section.

The contribution of the capillary layer to the rate of discharge has been estimated in equation (1). The factor 2 in this equation compares whit a value of 1 suggested by Muskat [3].
More extensive investigation may point to an intermediate value.

\section{D) Base Piezometric Head}

Yang's work [7] and the results of the present investigation indicate that the Dupuit-Thiem equation is a satisfactory approximation of the curve of base piezometric head, although the correspondence is perhaps not as close as earlier investigators have been led to conclude.

\section{E) Degree and Uniformity of Saturation}

It is concluded that degree of saturation is not a significant factor in affecting the flow pattern; but that degree of uniformity in the distribution of air voids is of great importance.

\section{F) Rate of Discharge}

Other investigators have already demonstrated the validity of the Dupuit-Thiem equation for computing the rate of discharge or average coefficient of permeability. The results of the present investigation provide additional data in support of this conclusion.

\section{ACKNOWLEDGEMENTS}

This paper is based on a dissertation submitted by the author to the Faculty of Arts and Sciences of Harvard University in partial fulfillment of the requirements for the degree of Doctor of Science in the field of Civil Engineering. In this connection the author wishes to make the following acknowledgements : To Professor Arthur Casagrande, for suggesting the problem and for his guidance throughout the investigation; to Professor S. D. Wilson, for assistance and sug- gestions in both the theoretical and experimental phases of the project; to Professor Harold A. Thomas, for several helpful suggestions; to Messrs. P. Grotjohan and R. Olsson, for valuable assistance in the construction of the model; and to the Soil Mechanics Research Fund of Harvard University for the necessary funds. The drawings for the present paper are by Mrs. Margaret M. Neigh.

\section{Bibliography (Bibliographie)}

[1] H. E. Babbitt and D. H. Caldwell, "The Free Surface around, and Interference between, Gravity Wells," Engineering Experiment Station, Bulletio Series, No. 374, University of Illinois, 1948.

[2] J. DupuIt, "Etudes théoriques et pratiques sur le mouvement des caux," Paris, 1863. (Second Edition.)

[3] M. Muskat, "Flow of Homogeneous Fluids through Porous Media," McGraw-Hill, 1937 (also Edwards, 1945).

[4] R. V. Southwell, "Relaxation Methods in Engineering Science," Oxford University Press, 1940.

[5] R. V. SouthwelL, "Relaxation Methods in Theoretical Physics," Oxford University Press, 1946.
[6] L. K. Wenzel, "Specific Yield Determined from A. Thiem's Pumping Test," Transactions of the American Geophysical Union, 13th Annual Meeting, 1932.

[7] S. T. YANG, "Seepage Toward a Well Analyzed by the Relaxation Method," Doctoral dissertation, Harvard University, 1949 (unpublished).

[8] N.S. Boulton, "The Flow Pattern Near a Gravity Well in a Uniform Water-Bearing Medium," Journal, Institution of Civil Engineers, Vol. 36, No. 10, December, 1951, pp. 534-550.

[9] V.E. Hansen, "Unconfined Ground-Water Flow to Multiple Wells," Transactions, American Society of Civil Engineers, Vol. 118, 1953, pp. 1098-1130. 